\title{
Pieri Type Rules and GL(2|2) Tensor Products
}

\section{Thorsten Heidersdorf ${ }^{1,2,3}$ (D) . Rainer Weissauer ${ }^{4}$}

Received: 28 May 2019 / Accepted: 6 February 2020 / Published online: 19 March 2020

(C) The Author(s) 2020

\begin{abstract}
We derive a closed formula for the tensor product of a family of mixed tensors using Deligne's interpolating category $\operatorname{Rep}\left(G L_{0}\right)$. We use this formula to compute the tensor product of a family of irreducible $\overline{G L(n} \mid n)$-representations. This includes the tensor product of any two maximal atypical irreducible representations of $G L(2 \mid 2)$.
\end{abstract}

Keywords Deligne categories · Representation of supergroups ·

Tensor product decomposition

Mathematics Subject Classification (2010) 17B10 · 17B20

\section{Introduction}

For the classical group $G L(n)$ the tensor product decomposition

$$
L(\lambda) \otimes L(\mu)=\bigoplus_{v} c_{\lambda \mu}^{\nu} L(v)
$$

between two irreducible representations is given by the Littlewood-Richardson rule for the Littlewood-Richardson coefficients $c_{\lambda \mu}^{\nu}$. Contrary to this case the analogous decomposition between two irreducible representation of the General Linear Supergroup $G L(m \mid n)$ is poorly understood. A classical result from Berele and Regev [1] and Sergeev [21] shows that the fusion rule between direct summands of tensor powers $V^{\otimes r}$ of the standard representation $V \simeq k^{m \mid n}$ is again given by the Littlewood-Richardson rule. The first more general

Presented by: Peter Littelmann

Thorsten Heidersdorf

heidersdorf.thorsten@gmail.com

Rainer Weissauer

weissauer@mathi.uni-heidelberg.de

1 Department of Mathematics, The Ohio State University, Columbus, OH, USA

2 Max-Planck Institut für Mathematik, Bonn, Germany

3 Institut für Mathematik, Universität Bonn, Bonn, Germany

4 Mathematisches Institut, Universität Heidelberg, Heidelberg, Germany 
results were achieved in [13] where we obtained a decomposition law for tensor products between any two mixed tensors, direct summands in a mixed tensor space $V^{\otimes r} \otimes\left(V^{\vee}\right)^{\otimes s}$, $r, s \in \mathbb{N}$. This result is based on the tensor product decomposition in Deligne's interpolating category $\operatorname{Rep}\left(G L_{\delta}\right)$ [8]. Due to the universal property of Deligne's category, we have for

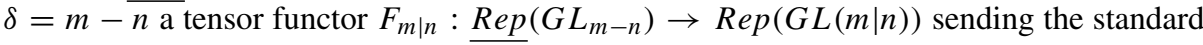
representation of the Deligne category to the standard representation $V=k^{m \mid n}$ of $G L(m \mid n)$. Since the decomposition of the tensor product of two indecomposable elements is known for $\operatorname{Rep}\left(G L_{m-n}\right)$ by results of Comes and Wilson [7], we obtain an analogous decomposition law once we describe the image $F_{m \mid n}(X)$ of an arbitrary indecomposable object $X$ in $\operatorname{Rep}\left(G L_{m-n}\right)$. This was achieved in [13] based on results by Brundan and Stroppel [6] on the interplay between Khovanov algebras and Walled Brauer algebras. Since any Kostant module [3] and any projective representation is a mixed tensor (up to some Berezin twist) [13], these results give a decomposition law for their tensor products, covering in particular the decomposition between any two irreducible $G L(m \mid 1)$-representations.

\subsection{The Main Results}

For $m, n \geq 2$ the irreducible mixed tensors are rather special. For example no non-trivial maximal atypical irreducible representation of $G L(n \mid n)$ is a mixed tensor. It is well-known that the weight of a maximal atypical representation is of the form $\lambda=\left(\lambda_{1}, \ldots, \lambda_{n} \mid-\right.$ $\left.\lambda_{n}, \ldots,-\lambda_{1}\right)$, and we denote the corresponding irreducible representation by $\left[\lambda_{1}, \ldots, \lambda_{n}\right]$. We also denote the irreducible representation $[i, 0, \ldots, 0]$ by $S^{i}$ for $i \geq 0$. In this paper we obtain an almost complete picture for the tensor product $S^{i} \otimes S^{j}$ for any $i, j$ and any $n$ and show

$$
S^{i} \otimes S^{j} \cong \delta_{i j} \text { Ber }^{i-1} \oplus M_{i j} \oplus \text { semisimple part }
$$

where $M_{i j}$ is a maximal atypical indecomposable representation and where the semisimple part is of atypicality $n-2$ and can be explicitely understood in terms of the decomposition law for $G_{0}$. This is the only known case of a formula apart from the $G L(m \mid 1)$-case and the case of Kostant modules.

Our interest in this result comes from different sources.

(1) In recent years the structure of $\mathcal{T}_{n}$ as an abelian category was determined in [5]. While other questions remain (e.g. an analogue of Borel-Weil-Bott, a more convenient character formula etc.), we view the description of the monoidal properties of $\mathcal{T}_{n}$ or its analogues for the other supergroups as one of the central questions in the theory. We hope that these results shed some light on this difficult and important problem.

(2) The Lie superalgebra $\mathfrak{g l}(2 \mid 2)$ or its simple counterpart $\mathfrak{p s l}(2 \mid 2)$ occurs in several physical models of AdS theory $[18,19]$. In fact similar formulas for the fusion rules have been obtained before in the more restrictive $\mathfrak{p s l}(2 \mid 2)$-case in the physics literature [12].

(3) The quotient of $\mathcal{T}_{n}=\operatorname{Rep}(G L(n \mid n))$ by its largest proper tensor ideal $\mathcal{N}$ is the representation category of a supergroup scheme $[15,16]$. The fusion rules obtained in this paper for $S^{i} \otimes S^{j}$ for $n=2$ play a crucial role in the determination of this group. More precisely, for every irreducible representation $L(\lambda)$ of non-vanishing superdimension we consider the tensor subcategory generated by it in $\mathcal{T}_{n} / \mathcal{N}$. It is equivalent to the representation category of a classical group $H_{\lambda}$ [15]. In fact these groups (or their connected derived groups) can be understood inductively starting with the case $n=2$ considered in this paper. The inductive determination rests then on the super 
tannakian formalism of Deligne and dimension and rank estimates. In this sense the case $n=2$ is harder than the higher rank cases for $n \geq 3$.

\subsection{Summary of the Proof}

While the article uses a fair amount of computation, its approach is rather conceptual and uses a lot of theory: Deligne's interpolating categories $\operatorname{Rep}\left(G L_{m-n}\right)$, the description of the functor $F_{n}: \operatorname{Rep}\left(G L_{0}\right) \rightarrow \operatorname{Rep}(G L(n \mid n))$, the knowledge of the Loewy layers of mixed tensors based on Brundan and Stroppels results about the connection between the walled Brauer algebra and Khovanov algebras [6] and last but not least the formalism of cohomological tensor functors and Tannaka groups of $[14,15]$. Let us describe the necessary steps.

\subsubsection{Step 1: The Deligne Category $\operatorname{Rep}\left(G L_{\delta}\right)$}

A first input is the tensor product decomposition in the Deligne category $\operatorname{Rep}\left(G L_{0}\right)$ $[7,8]$. The information about the tensor product decomposition between indecomposable objects in $\operatorname{Rep}\left(G L_{0}\right)$ can be transferred to $\mathcal{T}_{n}$ by means of the symmetric monoidal functor $F_{n \mid n}: \overline{\operatorname{Rep}}\left(G L_{0}\right) \rightarrow \operatorname{Rep}(G L(n \mid n))$ sending the standard representation of the Deligne category to the standard representation $V=k^{n \mid n}$ of $G L(n \mid n)$. This functor can be described explicitely as in [7, 13]. Implicitely results about the representation theory of the walled Brauer algebra play a crucial role here.

\subsubsection{Step 2: Khovanov Algebras}

The image $F_{n \mid n}(R(\lambda))$ of an indecomposable object $R(\lambda) \in \operatorname{Rep}\left(G L_{0}\right)$ can be determined [13] based on a description of the Loewy structure of direct summands in a mixed tensor space $V^{\otimes r} \otimes\left(V^{\vee}\right)^{\otimes s}[6]$. The results of Brundan and Stroppel are based on the combinatorial description of the blocks in $\mathcal{R}_{n}$ by means of the diagram algebra $K(m \mid n)$, a so-called Khovanov algebra [5]. The Loewy layers and the composition factors of the $F_{n \mid n}(R(\lambda))$ admit then a description in terms of the cup/cap combinatoric of the Khovanov algebras or, in other words, parabolic Kahzdan-Luztig theory for a maximal parabolic in type $A$.

\subsubsection{Step 3: The Modules $\mathbb{A}_{\boldsymbol{s}^{i}}$}

None of the irreducible representations $S^{i}$ is of the form $F_{n \mid n}(R(\lambda))$ (except for the trivial case $S^{0} \cong \mathbf{1}$ ). To circumvent this problem, we first consider special mixed tensors $\mathbb{A}_{S^{i}}$ which contain the irreducible representation $S^{i}$ as the constituent of highest weight with multiplicity 1. As all mixed tensors these are of the form $F_{n \mid n}(R(\lambda))$ [7]. We proceed as follows: We derive a closed formula for the tensor product decomposition $\mathbb{A}_{S^{i}} \otimes \mathbb{A}_{S^{j}}$. This computation takes place in $\underline{\operatorname{Rep}}\left(G L_{0}\right)$ and is then pushed to $\mathcal{T}_{n}$ via $F_{n \mid n}$.

\subsubsection{Step 4: $K_{0}$-Decomposition and the Case $n=2$}

The composition factors and the socle filtration of the $\mathbb{A}_{S^{i}}$ are known [13]. The tensor product $\mathbb{A}_{S^{i}} \otimes \mathbb{A}_{S^{j}}$ splits into representations of the form $\mathbb{A}_{S^{k}}$ for some $k$, maximal atypical mixed tensors $R(a, b)$ and a semisimple part which is not maximal atypical. We specialize now to 
the case $n=2$ and view this decomposition in the Grothendieck ring $K_{0}\left(\mathcal{T}_{2}\right)$ and derive from this a closed formula for $S^{i} \otimes S^{j}$ in the Grothendieck ring. The difficult part here is to understand the maximal atypical part since the other summands of lower atypicality are irreducible. In fact we can easily derive a general formula for the non maximal atypical part in $S^{i} \otimes S^{j}$ for any $n$ as in Section 7: The remaining composition factors in $\mathbb{A}_{S^{i}} \otimes \mathbb{A}_{S^{j}}$ are all $(n-2)$-fold atypical and it is easy to see that they always lie in different blocks. Hence they cannot combine to an indecomposable representation and the $K_{0}$-decomposition is already enough for the computation. The reason for the specialization to $n=2$ is that we need an explicit description of the composition factors of the maximal atypical indecomposable mixed tensors $R(a, b)$. While this is theoretically possible by step Section 1.2.2, the combinatorics becomes very complex for $n \geq 3$. In the $n=2$ these modules are projective covers and their composition factors can be described easily.

We split the computation of $S^{i} \otimes S^{j}$ into two parts. We first project onto the maximal atypical block $\Gamma$ and then compute the remaining summands afterwards in Section 7 . In

$$
\left[\mathbb{A}_{S^{i}}\right] \otimes\left[\mathbb{A}_{S^{j}}\right] \in K_{0}\left(\mathcal{R}_{2}\right)
$$

the tensor product $S^{i} \otimes S^{j}$ occurs exactly once, and all other tensor products are of the form $\operatorname{Ber}^{p}\left(S^{k} \otimes S^{l}\right)$ with both $k$ and $l$ less or equal to $i$ and $j$ and some Berezin power $p$. This allows us to compute the maximal atypical composition factors of $S^{i} \otimes S^{j}$ recursively in Lemma 5.2.

\subsubsection{Step 5: Cohomological Tensor Functors for $\boldsymbol{n}=2$}

In order to determine the decomposition of $S^{i} \otimes S^{j}$ into maximal atypical indecomposable representations we use the theory of cohomological tensor functors [14]. Here we consider the tensor functor $D S: \operatorname{Rep}(G L(2 \mid 2)) \rightarrow \operatorname{Rep}(G L(1 \mid 1))$. The main theorem of [14] gives a formula for $D S(L)$ for any irreducible representation and we get $D S\left(S^{i}\right)=B_{e r}{ }^{i} \oplus \Pi^{1-i} \mathrm{Ber}^{-1}$ where $\Pi$ denotes the parity shift functor. This gives us strict estimates on the number of indecomposable summands and their superdimension which is enough to determine the indecomposable summands in Theorem 5.7.

The steps 1 - 5 settle the entire $n=2$ case as well as the computation of the summands which are not maximal atypical for any $n$. The general case for $n \geq 3$ now follows rather easily assuming the formalism developed in [15]. Therefore some results in Section 6 depend on [15].

\subsubsection{Step 6: Reduction to the $n=2$-Case}

We show in Lemma 6.4 that the $S^{i} \otimes S^{j}$ decomposition is always clean: every maximal atypical summand has non-vanishing superdimension. This means that we can the see the decomposition behaviour in the quotient of $\mathcal{T}_{n}$ by the ideal of negligible morphisms. But the structure of this quotient has been exactly determined in [15] using the fusion rules for $n=2$ ! We stress that we need very little from the general setup of [15] to deal with the $S^{i}$ case. In fact the $S^{i}$ case can be completely separated from the remaining cases as shown in [15, Section 9.3].

These methods allow in principle to compute also the maximal atypical composition factors in the decomposition $S^{i} \otimes S^{j}$ for $n \geq 3$. However it is difficult to determine the composition factors of the maximal atypical mixed tensors $R(a, b)$ for $n \geq 3$. We end the article with a conjecture for the socle of $S^{i} \otimes S^{j}$ for arbitrary $n$. 


\section{The Superlinear Groups}

\subsection{Representations}

Let $k$ be an algebraically closed field of characteristic zero. Let $\mathfrak{g}=\mathfrak{g l}(n \mid n)=\mathfrak{g}_{0} \oplus \mathfrak{g}_{1}$ be the general linear superalgebra and $G L(n \mid n)$ the general linear supergroup. By definition a finite dimensional super representation $\rho$ of $\mathfrak{g l}(n \mid n)$ defines a representation $\rho$ of $G L(n \mid n)$ if its restriction to $\mathfrak{g}_{0}$ comes from an algebraic representation of $G_{0}=G L(n) \times G L(n)$, also denoted $\rho$. We denote the category of finite-dimensional representations with paritypreserving morphisms by $\mathcal{T}_{n}=\mathcal{T}_{n \mid n}$. For $M \in \mathcal{T}$ we denote by $M^{\vee}$ the ordinary dual and by $M^{*}$ the twisted dual. For simple and for projective objects $M$ of $\mathcal{T}$ we have $M^{*} \cong M$ [2].

\subsection{The Category $\mathcal{R}_{n}$}

Fix the morphism $\varepsilon: \mathbb{Z} / 2 \mathbb{Z} \rightarrow G_{0}=G L(n) \times G L(n)$ which maps -1 to the element $\operatorname{diag}\left(E_{n},-E_{n}\right) \in G L(n) \times G L(n)$ denoted $\epsilon_{n n}$. We write $\epsilon_{n}=\epsilon_{n n}$. Note that $\operatorname{Ad}\left(\epsilon_{n n}\right)$ induces the parity morphism on the Lie superalgebra $\mathfrak{g l}(n \mid n)$ of $G$. We define the abelian subcategory $\mathcal{R}_{n}$ of $\mathcal{T}_{n}$ as the full subcategory of all objects $(V, \rho)$ in $\mathcal{T}_{n}$ with the property $p_{V}=\rho\left(\epsilon_{n n}\right)$; here $\rho$ denotes the underlying homomorphism $\rho: G L(n) \times G L(n) \rightarrow$ $G L(V)$ of algebraic groups over $k$ and $p_{V}$ the parity automorphism of $V$. The subcategory $\mathcal{R}_{n}$ is stable under the dualities ${ }^{\vee}$ and ${ }^{*}$. The irreducible representations in $\mathcal{R}_{n}$ are indexed by dominant integral weights with respect to the standard Borel subalgebra of upper triangular matrices. We denote by $L(\lambda)$ the irreducible representation with highest weight $\lambda=\left(\lambda_{1}, \ldots, \lambda_{n} \mid \lambda_{n+1}, \ldots, \lambda_{2 n}\right)$ where $\lambda \in \mathbf{Z}^{2 n}$ is any element satisfying

$$
\lambda_{1} \geq \lambda_{2} \geq \ldots \geq \lambda_{n} \text { and } \lambda_{n+1} \geq \lambda_{n+2} \geq \ldots \geq \lambda_{2 n} .
$$

The Berezin determinant of $G L(n \mid n)$ defines a one dimensional representation $B=B e r$ with weight $(1, \ldots, 1 \mid-1, \ldots,-1)$. For each representation $M \in \mathcal{R}_{n}$ we also have its parity shifted version $\Pi(M)$ in $\mathcal{T}_{n}$. Since we only consider parity preserving morphisms, these two are not isomorphic. In particular the irreducible representations in $\mathcal{T}_{n}$ are given by the $\left\{L(\lambda), \Pi L(\lambda) \mid \lambda \in X^{+}\right\}$. The whole category $\mathcal{T}_{n}$ decomposes as $\mathcal{T}_{n}=\mathcal{R}_{n} \oplus$ $\Pi \mathcal{R}_{n}$ [2, Corollary 4.44]. An object $M \in \mathcal{T}_{n}$ is called negligible, if it is the direct sum of indecomposable objects $M_{i}$ in $\mathcal{T}_{n}$ with superdimensions $\operatorname{sim}\left(M_{i}\right)=0$. The thick ideal of negligible objects is denotes $\mathcal{N}$ or $\mathcal{N}_{n}$.

\subsection{Atypicality}

If $L(\lambda)$ is projective, the weight $\lambda$ is called typical. If not, $\lambda$ is called atypical. The atypicality of a weight can be measured by a number between 0 and $n$ [17]. If the atypicality is $n$, we say the weight is maximal atypical. An example is the Berezin determinant $\mathrm{Ber}$ of dimension 1. More generally an irreducible representation is maximal atypical if and only if $\lambda$ is of the form

$$
\lambda=\left(\lambda_{1}, \ldots, \lambda_{n} \mid-\lambda_{n}, \ldots,-\lambda_{1}\right) .
$$

In this case we often write $\left[\lambda_{1}, \ldots, \lambda_{n}\right]$ for $L(\lambda)$. We define for $i \geq 0$

$$
S^{i}=[i, 0, \ldots, 0] \in \mathcal{R}_{n} .
$$

The superdimension of an irreducible representation is non-zero if and only if $L(\lambda)$ is maximal atypical $[20,22]$.The abelian categories $\mathcal{T}_{n}$ and $\mathcal{R}_{n}$ decompose into blocks and the degree of atypicality is a block-invariant. 


\section{Mixed Tensors}

The decompositon of $S^{i} \otimes S^{j}$ is obtained from the decomposition $\mathbb{A}_{S^{i}} \otimes \mathbb{A}_{S^{j}}$ where the $\mathbb{A}_{S^{i}}$ are mixed tensors. We review some facts about them.

\subsection{Indecomposable Representations and Combinatorics of Bipartitions}

Let $\mathcal{M T}$ denote the full subcategory of mixed tensors in $\mathcal{R}_{n}$ whose objects are direct sums of the indecomposable objects in $\mathcal{R}_{n}$ that appear in a decomposition $V^{\otimes r} \otimes\left(V^{\vee}\right)^{\otimes s}$ for some natural numbers $r, s \geq 0$, where $V \in \mathcal{R}_{n}$ denotes the standard representation. By [6, Theorem 8.19] and [7, Theorem 8.18] the indecomposable objects in $\mathcal{M T}$ are parametrized by $(n \mid n)$-cross bipartitions (see below). Let $R_{n}(\lambda)$ (or $R(\lambda)$ if the dependency on $n$ is clear) denote the indecomposable representation in $\mathcal{R}_{n}$ corresponding to the bipartition $\lambda=\left(\lambda^{L}, \lambda^{R}\right)$ under this parametrization. We sometimes write $R\left(\lambda^{L}, \lambda^{R}\right)$ to avoid brackets. To any bipartition we attach a weight diagram in the sense of [4], i.e. a labelling of the numberline $\mathbb{Z}$ according to the following dictionary. Put

$$
I_{\wedge}(\lambda):=\left\{\lambda_{1}^{L}, \lambda_{2}^{L}-1, \lambda_{3}^{L}-2, \ldots\right\} \quad \text { and } \quad I_{\vee}(\lambda):=\left\{1-\lambda_{1}^{R}, 2-\lambda_{2}^{R}, \ldots\right\} .
$$

Now label the integer vertices $i$ on the numberline by the symbols $\wedge, \vee, \circ, \times$ according to the rule

$$
\begin{cases}\circ & \text { if } i \notin I_{\wedge} \cup I_{\vee}, \\ \wedge & \text { if } i \in I_{\wedge}, i \notin I_{\vee}, \\ \vee & \text { if } i \in I_{\vee}, i \notin I_{\wedge}, \\ \times & \text { if } i \in I_{\wedge} \cap I_{\vee} .\end{cases}
$$

To any such data one attaches a cup-diagram as in [7, 6.3] or [4] and we define the following three invariants

$$
\begin{aligned}
r k(\lambda) & =\text { number of crosses } \\
d(\lambda) & =\text { number of cups } \\
k(\lambda) & =r k(\lambda)+d(\lambda) .
\end{aligned}
$$

Example 3.1 Let $\lambda=\left(3,1^{3}\right)$. Then its weight diagram is

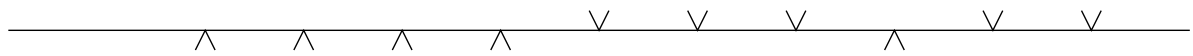

with the rightmost $\wedge$ at the vertex 3 . Its cup diagram is

Therefore $k(\lambda)=1, r k(\lambda)=0$ and $d(\lambda)=1$. For $\lambda=\left((4,2),\left(2^{2}, 1^{2}\right)\right)$ the weight diagram is

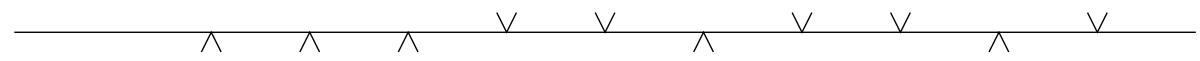

with the two rightmost $\wedge$ 's at the vertices 4 and 0 . Its cup diagram is

Therefore $k(\lambda)=2, r k(\lambda)=0$ and $d(\lambda)=2$. 
A bipartition is said to be $(n \mid n)$-cross if and only if $k(\lambda) \leq n$. By [6, Lemma 8.18] the modules $R\left(\lambda^{L}, \lambda^{R}\right)$ have irreducible socle and cosocle equal to $L\left(\lambda^{\dagger}\right)$ where the highest weight $\lambda^{\dagger}$ can be obtained by a combinatorial algorithm from $\lambda$. Let $\theta: \Lambda \rightarrow X^{+}(n)$ denote the resulting map $\lambda \mapsto \lambda^{\dagger}$ between the set of $(n \mid n)$-cross bipartitions $\Lambda$ and the set $X^{+}(n)$ of highest weights of $\mathcal{R}_{n}$.

Theorem 3.2 [13, Corollary 5.4, Theorem 5.12] $R=R\left(\lambda^{L}, \lambda^{R}\right)$ is an indecomposable module of Loewy length $2 d(\lambda)+1$. It is projective if and only if $k(\lambda)=n$ in which case we have $R=P\left(\lambda^{\dagger}\right)$. In particular $R(\lambda)$ is irreducible if $d(\lambda)=0$.

\subsection{The Map $\lambda \mapsto \lambda^{\dagger}$}

We recall the explicit description of the map $\theta$ as in [13, Section 6.1], i.e. we describe how to transform the weight diagram of the bipartition $\lambda$ into the weight diagram of the highest weight $\lambda^{\dagger}$. Define $M$ to be the largest vertex labelled with a $\times$ or $\circ$ or part of a cup in the weight diagram of $\lambda$ and put

$$
T=\max (k(\lambda)+1, M+1) .
$$

Now define

$$
X= \begin{cases}0 & M+1 \leq k(\lambda)+1 \\ M-k(\lambda) & \text { else. }\end{cases}
$$

We say a vertex is free if it does not have a cross, or a circle or is not part of a cup.

Corollary 3.3 [13, Corollary 6.3] The weight diagram of $\lambda^{\dagger}$ is obtained from the weight diagram of $\lambda$ by switching all $\vee$ 's to $\wedge$ 's and vice versa at vertices $\geq T$ and switching all $\vee$ 's to $\wedge$ 's and vice versa at the first $X+n-k(\lambda)$ free vertices $<T$.

\subsection{Deligne's Interpolating Category}

For every $\delta \in k$ we denote by $\operatorname{Rep}\left(G L_{\delta}\right)$ the interpolating category defined in [8]. This is a $k$-linear pseudoabelian rigid symmetric monoidal category. By construction it contains an object $s t$ of dimension $\delta$, called the standard representation. By the universal property [8, Proposition 10.3] of the Deligne category we have a tensor functor $F_{n}=F_{n \mid n}$ : $\operatorname{Rep}\left(G L_{0}\right) \rightarrow \mathcal{R}_{n}$ mapping the standard representation of $\operatorname{Rep}\left(G L_{0}\right)$ to the standard representation of $G L(n \mid n)$ in $\mathcal{R}_{n}$. Every mixed tensor is in the image of this tensor functor [7, 8.13]. The indecomposable objects in $\operatorname{Rep}\left(G L_{\delta}\right)$ are parametrized by bipartitions [7] and we denote by $R(\lambda)$ the indecomposable element associated to the bipartition $\lambda$. Then $[7,8.3]$

$$
F_{n}: R(\lambda) \mapsto \begin{cases}R_{n}(\lambda) & \text { if } k(\lambda) \leq n \\ 0 & \text { if } k(\lambda)>n .\end{cases}
$$

The atypicality of $R_{n}(\lambda)$ is given by $n-r k(\lambda)$ [13]. Note that the superdimension of every nontrivial mixed tensor vanishes since $\operatorname{sim}(V)=0$.

\section{The Symmetric and Alternating Powers}

We define as in [13] the following indecomposable modules in $\mathcal{R}_{n}$

$$
\mathbb{A}_{S^{i}}=R\left(i ; 1^{i}\right) \text { and } \mathbb{A}_{\Lambda^{i}}=\left(\mathbb{A}_{S^{i}}\right)^{\vee}=R\left(1^{i} ; i\right) .
$$


The aim of this section is to prove prove a formula for $\mathbb{A}_{S^{i}} \otimes \mathbb{A}_{S^{j}}$ in $\mathcal{R} \backslash$ by calculations in $\operatorname{Rep} G L_{0}$. We neglect in this section summands that are not maximal atypical.

\subsection{Loewy Layers and the $G L(1 \mid 1)$-Case}

Recall that we defined $S^{i}=[i, 0, \ldots, 0]$ for integers $i \geq 1$. We denote the trivial representation $S^{0}$ by $\mathbf{1}$. Furthermore we denote the projective cover of $[\lambda]$ by $P[\lambda]$.

Lemma 4.1 [13, Lemma 13.3] The Loewy structure of the $\mathbb{A}_{S^{i}}$ is given by $(n \geq 2)$

$$
\begin{aligned}
& \mathbb{A}_{S^{1}}=\left(\mathbf{1}, S^{1}, \mathbf{1}\right) \\
& \mathbb{A}_{S^{i}}=\left(S^{i-1}, S^{i} \oplus S^{i-2}, S^{i-1}\right) \quad 1<i \neq n \\
& \mathbb{A}_{S^{n}}=\left(S^{n-1}, S^{n} \oplus S^{n-2} \oplus B^{-1}, S^{n-1}\right) .
\end{aligned}
$$

In particular $S^{i}$ is the constituent of highest weight in $\mathbb{A}_{S^{i}}$.

We remark that mixed tensors are always rigid [13, Corollary 5.4]. These representations are maximal atypical for any $n$. We now derive a closed formula for the tensor products $\mathbb{A}_{S^{i}} \otimes \mathbb{A}_{S^{j}}$. It turns out that the maximal atypical summands are not irreducible whereas all other summands are irreducible. Therefore we split the computations in two parts: we first compute the projection to the maximal atypical block of $\mathbb{A}_{S^{i}} \otimes \mathbb{A}_{S^{j}}$ and deal with the remaining easier case later in Section 7. In the following formulas we often project to the maximal atypical block. Recall from [13, Proposition 11.1] that a mixed tensor $R\left(\lambda^{L}, \lambda^{R}\right)$ is maximal atypical if and only if $\lambda^{R}=\left(\lambda^{L}\right)^{*}$ where $\lambda^{*}$ denotes the conjugate partition. In this case we simply use the notation $R\left(\lambda^{L}\right)$, e.g. $\mathbb{A}_{S^{i}}=R(i)$ and $\mathbb{A}_{\Lambda^{i}}=R\left(1^{i}\right)$.

Lemma 4.2 The atypical mixed tensors in $\mathcal{R}_{1}$ are the $\mathbb{A}_{S^{i}}$ and their duals $\mathbb{A}_{\Lambda^{j}}$. They are the projective covers $\mathbb{A}_{S^{i}}=P[i-1]$ and $\mathbb{A}_{\Lambda^{j}}=P[-j+1]$.

Proof Any mixed tensor $R(\lambda)$ in $\mathcal{R}_{1}$ satisfies $k(\lambda) \leq 1$. If $k(\lambda)=0$, then $R(\lambda)$ is irreducible and singly atypical and therefore $R(\lambda) \cong \mathbf{1}$. If $k(\lambda)=1$, then it is projective by Theorem 3.2. If $r k(\lambda)=1, R(\lambda)$ is typical. The statement about the top follows from an explicit computation of the map $\theta: \Lambda \rightarrow X^{+}[13,6.1]$ already done in the proof of [13, Lemma 13.3].

Corollary 4.3 In $\mathcal{R}_{1}$

$$
\begin{aligned}
\mathbb{A}_{S^{i}} \otimes \mathbb{A}_{\Lambda^{j}} & =\mathbb{A}_{S^{|-i+j|+2}} \oplus 2 \mathbb{A}_{S^{|-i+j|+1}} \oplus \mathbb{A}_{S^{|-i+j|}} \\
\mathbb{A}_{S^{i}} \otimes \mathbb{A}_{S^{j}} & =\mathbb{A}_{S^{i+j}} \oplus 2 \cdot \mathbb{A}_{S^{i+j-1}} \oplus \mathbb{A}_{S^{i+j-2}}
\end{aligned}
$$

Proof This is just rewriting the known formula $(a, b \in \mathbf{Z})$

$$
P[a] \otimes P[b]=P[a+b+1] \oplus 2 P[a+b] \oplus P[a+b-1]
$$

from [11].

Let us assume from now on that $n \geq 2$. 
Lemma 4.4 After projection to the maximal atypical block $(n \geq 2)$

$$
\begin{aligned}
& \mathbb{A}_{S^{i}} \otimes \mathbb{A}_{\Lambda^{j}}=\mathbb{A}_{S^{|-i+j|+2}} \oplus 2 \mathbb{A}_{S^{|-i+j|+1}} \oplus \mathbb{A}_{S^{|-i+j|}} \oplus R_{1} \\
& \mathbb{A}_{S^{i}} \otimes \mathbb{A}_{S^{j}}=\mathbb{A}_{S^{i+j}} \oplus 2 \cdot \mathbb{A}_{S^{i+j-1}} \oplus \mathbb{A}_{S^{i+j-2}} \oplus R_{2}
\end{aligned}
$$

where $R_{1}$ and $R_{2}$ are direct sums of modules which do not contain any $\mathbb{A}_{S^{i}}$ or $\mathbb{A}_{\Lambda^{j}}$.

Proof This follows from the $G L(1 \mid 1)$-case and the identification between the projective covers and the symmetric and alternating powers. In $G L(1 \mid 1)[11]$

$$
P[a] \otimes P[b]=P[a+b-1] \oplus 2 P[a+b] \oplus P[a+b+1] .
$$

Hence this formula holds for the corresponding $\mathbb{A}_{S^{i}}$ respectively $\mathbb{A}_{\Lambda^{j}}$. It then holds in $\operatorname{Rep}\left(G L_{0}\right)$ up to summands in the kernel of the functor $F_{1}: \operatorname{Rep}\left(G L_{0}\right) \rightarrow \operatorname{Rep}(G L(1 \mid 1))$ of Section 3. The kernel consists of the $R(\lambda)$ with $k(\lambda)>1$. By [13, Lemma 13.1] a maximal atypical mixed tensor satisfies $d(\lambda)=1$ (and hence $k(\lambda)=1$ ) if and only if and only if $\lambda=\left(i ; 1^{i}\right)$ or $\lambda=\left(1^{i} ; i\right)$. Hence this formula holds in any $\operatorname{Rep}(G L(n \mid n))$ up to contributions which lie in the kernel of $F_{n \mid n}: \underline{\operatorname{Rep}}\left(G L_{0}\right) \rightarrow \operatorname{Rep}(G L(n \mid n))$ and which are not (1|1)-cross.

\subsection{Tensor Products in Deligne's Category}

In order to compute $\mathbb{A}_{S^{i}} \otimes \mathbb{A}_{S^{j}}$ in $\mathcal{R}_{n}$ we compute $R(i) \otimes R(j)$ in $\operatorname{Rep}\left(G L_{0}\right)$. We then push the result to $\operatorname{Rep}(G L(n \mid n))$ using $F_{n}$. We recall the tensor product decomposition in $\underline{\operatorname{Rep}}\left(G L_{0}\right)$.

\subsubsection{The Lifting Map}

We attach to the weight diagram of a bipartition a cap-diagram as in $[4,7]$. We denote the degree $\sum_{i} \lambda_{i}$ of a partition by $|\lambda|$. If $|\lambda|=n$ we write $\lambda \vdash n$. If $\lambda=\left(\lambda^{L}, \lambda^{R}\right)$ is a bipartition we denote its degree $\left(\left|\lambda^{L}\right|,\left|\lambda^{R}\right|\right)$ by $|\lambda|$ and we write $\lambda \vdash(r, s)$ if $\left|\lambda^{L}\right|=r$ and $\left|\lambda^{R}\right|=s$. Let us fix a bipartition $\lambda$ and consider the associated weight and cup diagram. For integers $i<j$ we say that $(i, j)$ is a $\vee \wedge$-pair if they are joined by a cap. For $\lambda, \mu \in \Lambda$ we say that $\mu$ is linked to $\lambda$ if there exists an integer $k \geq 0$ and bipartitions $v^{(n)}$ for $0 \leq n \leq k$ such that $v^{(0)}=\lambda, v^{(k)}=\mu$ and the weight diagramm of $v^{(n)}$ is obtained from the one of $v^{(n-1)}$ by swapping the labels of some pair $\vee \wedge$-pair. Then we put

$$
D_{\lambda, \mu}= \begin{cases}1 & \mu \text { is linked to } \lambda \\ 0 & \text { otherwise. }\end{cases}
$$

Then one has $D_{\lambda, \lambda}=1$ for all $\lambda$. Further $D_{\lambda, \mu}=0$ unless $\mu=\lambda$ or $|\mu|=\left(\left|\lambda^{L}\right|-i,\left|\lambda^{R}\right|-i\right)$ for some $i>0$ (see [7, Theorem 6.2.3] for further details).

Example 4.5 We saw in Example 3.1 that the cup diagram for $\lambda=\left((4,2),\left(2^{2}, 1^{2}\right)\right)$ is

Then there are 4 partitions linked to $\lambda: \lambda$ itself, 2 partitions obtained from $\lambda$ by interchanging the labels in one of the cups and a fourth summand by interchanging the labels in the two cups simultaneously.

Let $t$ be an indeterminate and $R_{\delta}$ respective $R_{\delta, t}$ the Grothendieck rings of $\operatorname{Rep}\left(G L_{\delta}\right)$ over $k$ respective of $\left.\underline{\operatorname{Rep}}\left(G L_{t}\right)\right)$ over the fraction field $k((t-\delta))$. We follow the notation 
of [7] and denote by ( $\lambda)$ or simply $\lambda$ the element $R(\lambda)$ in $R_{\delta, t}$ or $R_{\delta}$. Now define lift $\delta$ : $R_{\delta} \rightarrow R_{\delta, t}$ as the $\mathbf{Z}$-linear map defined by $\operatorname{lift}_{\delta}(\lambda)=\sum_{\mu} D_{\lambda, \mu} \mu$ where the sum runs over all bipartitions $\mu$. By [7, Theorem 6.2.3] lift $\delta$ is a ring isomorphism for every $\delta \in k$.

\subsubsection{Generic Tensor Product Decomposition}

By [7, Theorem 7.1.1] the following decomposition holds for arbitrary bipartitions in $R_{\delta, t}$ :

$$
\lambda \mu=\sum_{v \in P \times P} \Gamma_{\lambda \mu}^{v} v
$$

with the numbers

$$
\Gamma_{\lambda \mu}^{\nu}=\sum_{\alpha, \beta, \eta, \theta \in P}\left(\sum_{\kappa \in P} c_{\kappa \alpha}^{\lambda^{L}} c_{\kappa \beta}^{\mu^{R}}\right)\left(\sum_{\gamma \in P} c_{\gamma \eta}^{\lambda^{R}} c_{\gamma \theta}^{\mu^{L}}\right) c_{\alpha \theta}^{\nu^{L}} c_{\beta \eta}^{\nu^{R}},
$$

see [7, Theorem 5.1.2]. Here $c_{\lambda \mu}^{\nu}$ denotes the Littlewood-Richardson coefficient and $P$ the set of all partitions. In particular if $\lambda \vdash(r, s), \mu \vdash\left(r^{\prime}, s^{\prime}\right)$, then $\Gamma_{\lambda \mu}^{\nu}=0$ unless $|\nu| \leq$ $\left(r+r^{\prime}, s+s^{\prime}\right)$. So to decompose the tensor product $R(\lambda) \otimes R(\mu)$ in $\operatorname{Rep}\left(G L_{\delta}\right)$ apply the following three steps:

(1) Determine lift $\delta(\lambda \mu)$ in $R_{\delta, t}$,

(2) c use the formula for $\Gamma_{\lambda \mu}^{\nu}$ above to compute the decomposition in $R_{\delta, t}$

(3) and then take $\operatorname{lift}_{\delta}^{-1}$.

\subsection{Computations in $R_{t}$}

We continue to use our notation for the maximal atypical case and write $(i)$ instead of $\left(i ; 1^{i}\right)$. Clearly $\operatorname{lift}(i)=(i)+(i-1), \operatorname{lift}\left(1^{i}\right)=\left(1^{i}\right)+\left(1^{i-1}\right)$. We compute $R(i) \otimes R(j)$ in $\operatorname{Rep}\left(G L_{0}\right)$ following the three steps above. Hence in order to compute the tensor product $\overline{R(i)} \otimes R(j)$ we have to compute the tensor product $(i) \otimes(j)+(i) \otimes(j-1)+(i-1) \otimes$ $(j)+(i-1) \otimes(j-1)$ in $R_{\delta, t}$. We derive first a closed formula for $(i) \otimes(j)$ in $R_{t}$, i.e. for $\left((i, 0, \ldots),\left(1^{i}\right)\right) \otimes(j, 0, \ldots),\left(1^{j}\right)$.

- We analyze the sum $\sum_{\gamma \in P} c_{\gamma, \theta}^{\lambda^{R}} c_{\gamma, \eta}^{\mu^{L}}$. Here $\lambda^{R}=\left(1^{i}\right)$ and $\mu^{L}=(j, 0, \ldots)$. We need to find all pairs of partitions $(a, b)$ such that $c_{a, b}^{\mu^{L}}$ is non-zero. We denote this by $\left(\mu^{L}\right)^{-1}$. Now the Pieri rule gives $\left(\mu^{L}\right)^{-1}=(0, j),(1, j-1), \ldots,(j-1,1),(j, 0)$ and $\left(\lambda^{R}\right)^{-1}=\left(0,1^{i}\right),\left(1,1^{i-1}\right), \ldots,\left(1^{i}, 0\right)$. Hence $c_{\alpha, \theta}^{\lambda^{R}} c_{\beta, \eta}^{\mu^{L}}$ is zero unless $(\gamma, \theta)$ and $(\gamma, \eta)$ are of the form $(0, i)$ and $\left(0,1^{j}\right)$ or are of the form $(1, i-1)$ and $\left(1,1^{j-1}\right)$.

- The contribution $\sum_{\kappa \in P} c_{\kappa, \alpha}^{\lambda^{L}} c_{\kappa, \beta}^{\mu^{R}}$ : Here $\mu^{R}=\left(1^{j}\right), \lambda^{L}=(i)$. Similarly to the previous case this gives only the possibilities $c_{0, i}^{i} c_{0,1^{j}}^{1^{j}}$ and $c_{1, i-1}^{i} c_{1,1^{j-1}}^{1^{j}}$.

Hence the sum

$$
\sum_{\alpha, \beta, \eta, \theta}\left(\sum_{\kappa \in P} c_{\kappa, \alpha}^{\lambda^{L}} c_{\kappa, \beta}^{\mu^{R}}\right)\left(\sum_{\gamma \in P} c_{\gamma, \eta}^{\lambda^{R}} c_{\gamma, \theta}^{\mu^{L}}\right)
$$

collapses to

$$
\left(c_{0, i}^{i} c_{0,1^{j}}^{1^{j}}+c_{1, i-1}^{i} c_{1,1^{j-1}}^{1^{j}}\right)\left(c_{0,1^{i}}^{1^{i}} c_{0, j}^{j}+c_{1,1^{i-1}}^{1^{i}} c_{1, j-1}^{j}\right) .
$$

This corresponds to the choices 
- $\quad$ (A) $\alpha=i, \beta=1^{j}$

- (B) $\alpha=i-1, \beta=1^{j-1}$

- (C) $\eta=1^{i}, \theta=j$

- (D) $\eta=1^{i-1}, \theta=j-1$.

Only for these choices $A C, A D, B C, B D$ can there be a summand (v) with nonvanishing $\Gamma_{\lambda \mu}^{\nu}=c_{\alpha, \theta}^{\nu^{L}} c_{\beta, \eta}^{\nu^{R}}$.

Notation From now on we only consider bipartitions $v$ with $v^{L}=\left(v^{R}\right)^{*}$ and think of such a bipartition as a partition $v^{L}$. Only these bipartitions will give maximal atypical summands in $\mathcal{R}_{n}$. The other summands can be easily calculated later in Section 7.

- The AC-case: $c_{i, j}^{v^{L}} c_{1^{j}, 1^{i}}^{v^{R}}\left(v^{L}, v^{R}\right)$. By the Pieri rule $v^{L}$ can be any of $(i+j),(i+j-$ $1,1),(i+j-2,2), \ldots$ and $v^{R}$ any of $\left(1^{i+j}\right),\left(2,1^{i+j-2}, \ldots,(i,|i-j|)\right.$. Hence the following partitions $v$ (i.e. bipartitions of the form $\left(v^{L} ;\left(v^{L}\right)^{*}\right)$ appear with multiplicity 1:

$$
(i+j),(i+j-1,1), \ldots,((\max (i, j), \min (i, j)) .
$$

- The AD-case: $c_{i, j-1}^{v^{L}} c_{1^{j}, 1^{i-1}}^{v^{R}}$. Restricting to $v^{L}=\left(v^{R}\right)^{*}$ we obtain

$$
v \in\{(i+j-1),(i+j-2,1), \ldots,((\max (i, j), \min (i, j)-1))\} .
$$

- The BC-case: $c_{i-1, j}^{v^{L}} c_{1^{j-1}, 1^{i}}^{\nu^{R}}$. Here $v$ is any of

$$
v \in\{((i+j-1),(i+j-2,1), \ldots,((\max (i, j), \min (i, j)-1))\} .
$$

- The BD-case: $c_{i-1, j-1}^{v^{L}} c_{1^{j-1}, 1^{i-1}}^{v^{R}}$. Here

$$
v \in\{((i+j-2),(i+j-3,1), \ldots,(\max (i-1, j-1), \min (i-1, j-1)) .\}
$$

Hence in the Grothendieck ring $R_{\delta, t}$

$$
\begin{aligned}
& (i) \otimes(j)= \\
& (i+j)+(i+j-1,1)+\ldots+((\max (i, j), \min (i, j)) \\
& +(i+j-1)+(i+j-2,1)+\ldots+((\max (i, j), \min (i, j)-1)) \\
& +(i+j-1)+(i+j-2,1)+\ldots+((\max (i, j), \min (i, j)-1)) \\
& +((i+j-2)+(i+j-3,1)+\ldots+(\max (i-1, j-1), \min (i-1, j-1)) .
\end{aligned}
$$

\subsection{Going Back to $\operatorname{Rep}\left(G L_{0}\right)$}

We calculate now the inverse lift $^{-1}$ to get the decomposition in $\operatorname{Rep}\left(G L_{0}\right)$. In the special case $j=1, i>1$ we get $(j-1)=0$ and hence $\operatorname{lift}((i) \otimes(1)) \overline{=(i)} \otimes(1)+(i)+(i-$ $1)+(i-1) \otimes(1)$. In $R_{t}$ we have

$$
(i) \otimes(1)=(i+1)+(i, 1)+2(i)+(i-1)
$$

so that

$$
\operatorname{lift}((i) \otimes(1))=(i+1)+(i, 1)+4(i)+(i-1,1)+4(i-1)+(i-2) .
$$

After removing the contributions which will lead to $R(i+1) \oplus 2 R(i) \oplus R(i-1)$ we are left with $(i, 1)+(i)+(i-1,1)+(i-1)$. This is the lift of $(i, 1)$ and hence the indecomposable module $R(i, 1)$ appears as a direct summand. 
Lemma 4.6 In $\underline{\operatorname{Rep}}\left(G L_{0}\right)$ we have for $i \geq 2$

$$
R(i) \otimes R(1)=R(i+1) \oplus 2 R(i) \oplus R(i-1) \oplus R(i, 1) .
$$

In the general case we add up the contributions $((i)+(i-1)) \cdot((j)+(j-1))=$ $(i)(j)+(i)(j-1)+(i-1)(j)+(i-1)(j-1)$. All the summands are of the following types $(a, 0),(a, b), a>b>0$ or $(a, a), a>0$. We have

$$
\begin{aligned}
& \operatorname{lift}(a, b)=(a, b)+(a, b-1)+(a-1, b)+(a-1, b-1), \quad a>b>0 \\
& \operatorname{lift}(a, a)=(a, a)+(a, a-1)+(a-1, a-2)+(a-2, a-2) .
\end{aligned}
$$

After removing the contributions in $R_{\delta, t}$ which will give the $R(i+j) \oplus 2 R(i+j-$ 1) $\oplus R(i+j-2)$ and applying successively the liftings from above we get the following decompositions. For $i>2, j=2$ we get

$$
\begin{aligned}
R(i) \otimes R(2) & =R(i+2) \oplus 2 R(i+2) \oplus R(i) \\
& \oplus R(i+1,1) \oplus R(i, 2) \oplus 2 \cdot R(i, 1) \oplus R(i-1,1)
\end{aligned}
$$

Assume now $i>2, j \geq 2$ and $i>j$. Then

$$
\begin{aligned}
R(i) \otimes R(j) & =R(i+j) \oplus 2 R(i+j-1) \oplus R(i+j-2) \\
& \oplus R(i+j-1,1) \\
& \oplus R(i+j-2,2) \oplus 2 \cdot R(i+j-2,1) \\
& \oplus R(i+j-3,3) \oplus 2 \cdot R(i+j-3,2) \oplus R(i+j-3,1) \\
& \oplus R(i+j-4,4) \oplus 2 \cdot R(i+j-4,3) \oplus R(i+j-4,2) \\
& \oplus R(i+j-5,5) \oplus \ldots \\
& \oplus R(i, j) \oplus 2 \cdot R(i, j-1) \oplus R(i, j-2) \\
& \oplus R(i-1, j-1) .
\end{aligned}
$$

Now assume $i=j$. For $i=j=2$ we get

$$
\begin{aligned}
R(2) \otimes R(2) & =R(4) \oplus 2 R(3) \oplus R(2) \\
& \oplus R(3,1) \oplus R(2,2) \oplus 2 \cdot R(2,1) .
\end{aligned}
$$

For $i=j>2$ we get the same result as for $i \neq j$ while omitting the last factor $\oplus R(i-1, j-1)$.

Remark 4.7 In the same way one can compute a closed formula of the tensor product $R(i) \otimes$ $R\left(1^{j}\right)$. This is not needed for the $G L(2 \mid 2)$ calculations.

\section{GL(2|2) Tensor Products - the Maximal Atypical Part}

We compute the decomposition of the tensor product of any two maximal atypical irreducible modules in $\mathcal{R}_{2}$. In this section we compute only the direct summands which are maximal atypical. The remaining summands are computed in Section 7. The basic idea is to look at our formulas for $\mathbb{A}_{S^{i}} \otimes \mathbb{A}_{S^{j}}$ in the Grothendieck group and use these to compute the composition factors of $S^{i} \otimes S^{j}$ recursively starting with the obvious tensor product $S^{i} \otimes S^{0}$. We then determine the decomposition into indecomposable summands using results on cohomological tensor functors [14] and case-by-case distinctions. 


\subsection{The $\mathcal{R}_{2}$-Case: Setup}

Recall from Section 2.2 the Berezin determinant $B=\operatorname{Ber}=L(1, \ldots, 1 \mid-1, \ldots,-1)$. Its tensor powers $\mathrm{Ber}^{i}=L(i, \ldots, i \mid-i, \ldots,-i)$ are referred to as Berezin twists. In general

$$
B e r^{i} \otimes L\left(\lambda_{1}, \ldots, \lambda_{n} \mid \lambda_{n+1}, \ldots, \lambda_{2 n}\right)=L\left(\lambda_{1}+i, \ldots, \lambda_{n}+i \mid \lambda_{n+1}-i, \ldots, \lambda_{2 n}-i\right) .
$$

Every maximally atypical irreducible representation $L(\lambda)=\left[\lambda_{1}, \lambda_{2}\right]$ (in the notation of Section 2) is a Berezin twist of a representation of the form $S^{i}:=[i, 0]$ for $i \in \mathbb{N}$. Since tensoring with Ber is a flat functor, it is therefore enough to decompose the tensor product $S^{i} \otimes S^{j}$. The Ext-quiver of the maximal atypical block $\Gamma$ of $\mathcal{R}_{2}$ can be easily determined from [3]. It has been worked out by [9]. For all irreducible modules in $\Gamma$ we have $\operatorname{dim} \operatorname{Ext}^{1}(L(\lambda), L(\mu))=\operatorname{dim}_{\operatorname{Ext}}{ }^{1}(L(\mu), L(\lambda))=0$ or 1 . The Ext-quiver can be picturised as follows where a line segment between two irreducible modules denotes a nontrivial extension class between these two modules and where an irreducible module $[x, y]$ is represented as a point in $\mathbf{Z}^{2}$.

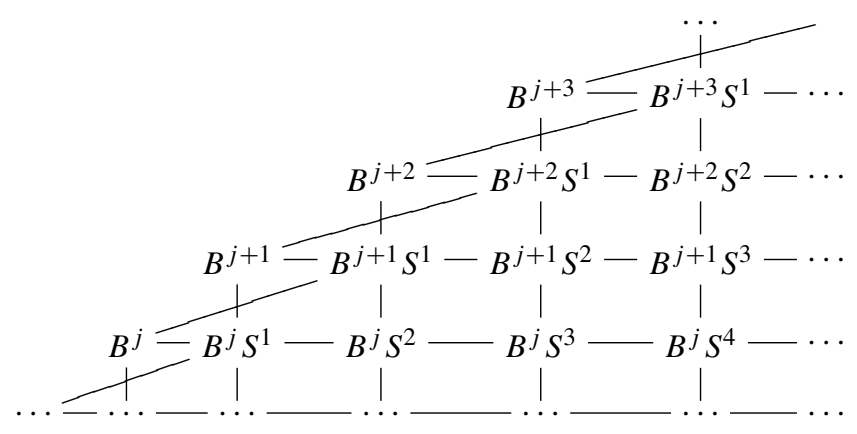

The Loewy structure of the projective covers of a maximally atypical irreducible module can also be computed from [5] or be taken from Drouot: For [a, $b], a=b+k, k \geq 3$ the Loewy structure (we display the socle layers) is

$$
P[a, b]=\left(\begin{array}{cccc}
B^{a-k} S^{k+1} & B^{a-k} S^{k-1} & B^{a-k-1} S^{k+1} & B^{a-k+1} S^{k-1} \\
2 B^{a-k} S^{k} & B^{a-k-1} S^{k+2} & B^{a-k-1} S^{k} & B^{a-k+2} S^{k-3} \\
B^{a-k} S^{k+1} & B^{a-k} S^{k-1} & B^{a-k-1} S^{k+1} & B^{a-k+1} S^{k-1}
\end{array}\right) .
$$

For $[a, b], a=b+2$ the Loewy structure is

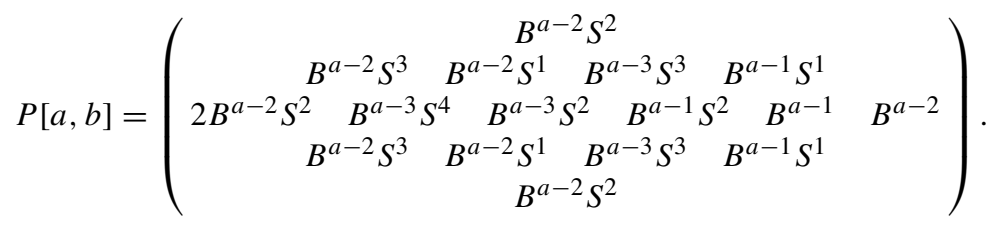

For $[a, b], a=b+1$ the Loewy structure is

$$
P[a, b]=\left(\begin{array}{ccccc}
B^{a-1} S^{2} & B^{a-1} & B^{a-2} S^{2} & B^{a} & B^{a-2} \\
2 B^{a-1} S^{1} & B^{a-2} S^{3} & B^{a-2} S^{1} & B^{a} S^{1} \\
B^{a-1} S^{2} & B^{a-1} & B^{a-2} S^{2} & B^{a} & B^{a-2}
\end{array}\right)
$$


For $[a, b], a=b$ the Loewy structure is

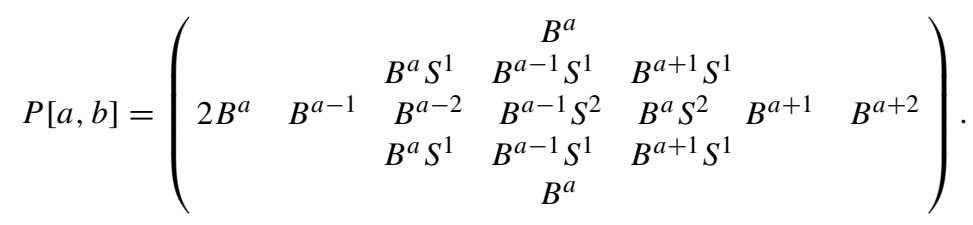

\subsection{The $\mathcal{R}_{2}$-Case: Mixed Tensors}

All direct summands in the decomposition $R(i) \otimes R(j)$ in $R e p G L_{0}$ satisfy $k(\lambda) \leq 2$. Hence they are not in the kernel of $F_{n \mid n}: \operatorname{Rep}\left(G L_{0}\right) \rightarrow \mathcal{R}_{n}$ for any $n \geq 2$. Therefore the formulas in the last section give us the maximal atypical summands in the decomposition of $\mathbb{A}_{S^{i}} \otimes \mathbb{A}_{S^{j}}$ for any $n \geq 2$. We specialise this decomposition to the $\mathcal{R}_{2}$-case. All formulas hold only after projection to $\Gamma$. It is easy to see that the $R(a, b)(b>0)$ satisfy $k(\lambda)=2$ and hence are projective covers of irreducible maximal atypical representations. The top and socle of these covers can be easily computed using the map $\theta: \Lambda \rightarrow X^{+}$(see Section 3.2). For small $j$ we get

$$
\begin{aligned}
\mathbb{A}_{S^{1}} \otimes \mathbb{A}_{S^{1}} & =\mathbb{A}_{S^{2}} \oplus 2 \cdot \mathbb{A}_{S^{1}} \oplus \mathbb{A}_{S^{2}}^{\vee} \\
\mathbb{A}_{S^{i}} \otimes \mathbb{A}_{S^{1}} & =\mathbb{A}_{S^{i+1}} \oplus 2 \cdot \mathbb{A}_{S^{i}} \oplus \mathbb{A}_{S^{i-1}} \oplus P[i-1,0] . \\
\mathbb{A}_{S^{i}} \otimes \mathbb{A}_{S^{2}} & =\mathbb{A}_{S^{i+2}} \oplus 2 \cdot \mathbb{A}_{S^{i+1}} \oplus \mathbb{A}_{S^{i}} \\
& \oplus P([i, 0]) \oplus P([i-1,1] \oplus 2 \cdot P([i-1,0]) \oplus P([i-2,0])
\end{aligned}
$$

where we assumed $i>1$ respectively $i>2$. Assume now $i>2, j \geq 2$ and $i>j$.

$$
\begin{aligned}
\mathbb{A}_{S^{i}} \otimes \mathbb{A}_{S^{j}} & =\mathbb{A}_{S^{i+j}} \oplus 2 \cdot \mathbb{A}_{S^{i+j-1}} \oplus \mathbb{A}_{S^{i+j-2}} \\
& \oplus P[i+j-2,0]) \\
& \oplus P[i+j-3,1] \oplus 2 \cdot P[i+j-3,0] \\
& \oplus P[i+j-4,2] \oplus 2 \cdot P[i+j-4,1] \oplus P[i+j-4,0] \\
& \oplus P[i+j-5,3] \oplus 2 \cdot P[i+j-5,2] \oplus P[i+j-5,1] \\
& \oplus P[i+j-6,4] \oplus \ldots \\
& \oplus P[i-1, j-1] \oplus 2 \cdot P[i-1, j-2] \oplus P[i-1, j-3] \\
& \oplus P[i-2, j-2] .
\end{aligned}
$$

For $i=j=2$

$$
\mathbb{A}_{S^{2}} \otimes \mathbb{A}_{S^{2}}=\mathbb{A}_{S^{4}} \oplus 2 \mathbb{A}_{S^{3}} \oplus \mathbb{A}_{S^{2}} \oplus P[2,0] \oplus P[0,0] \oplus 2 P[1,0] .
$$

For $i=j>2$ we have the same result without the last summand $P[i-2, j-2]$.

\subsection{The $\mathcal{R}_{2}$-Case: $K_{0}$-Decomposition}

The tensor product decomposition of the $\mathbb{A}_{S^{i}} \otimes \mathbb{A}_{S^{j}}$ along with the knowledge of the composition factors of the indecomposable summands permits to give recursive formulas for the $K_{0}$-decomposition of the tensor products $S^{i} \otimes S^{j}$ in the Grothendieck ring $K_{0}=K_{0}\left(\mathcal{R}_{n}\right)$. Due to the asymmetry of the formulas and the asymmetry of the $K_{0}$-decompositions for $\mathbb{A}_{S^{i}}$ and $P[a, b]$ for small $i$ and $a-b$ we compute the tensor products for small $i$ and $j$ first. 
The $K_{0}$-decomposition $S^{1} \otimes S^{1}$ follows immediately from the $\mathbb{A}_{S^{1}} \otimes \mathbb{A}_{S^{1}}$-decomposition and we get

$$
S^{1} \otimes S^{1}=2 \mathbf{1}+2 S^{1}+B+B^{-1}+B^{-1} S^{2}+S^{2} .
$$

Similarly one computes

$$
\begin{aligned}
& S^{2} \otimes S^{1}=2 S^{2}+S^{3}+B^{-1} S^{3}+S^{1}+B S^{1} \\
& S^{2} \otimes S^{2}=S^{4}+B^{-1} S^{4}+2 S^{3}+S^{2}+B S^{2}+2 B S^{1}+\mathbf{1}+2 B+B^{2} .
\end{aligned}
$$

Lemma 5.1 We have $P[i, 0]=2 \mathbb{A}_{S^{i+1}}+B^{-1} \mathbb{A}_{S^{i+2}}+B \mathbb{A}_{S^{i}}$ for $i \geq 1$ in $K_{0}\left(\mathcal{R}_{2}\right)$.

Proof This is just a direct inspection of the Loewy structures above.

Lemma 5.2 For all $i>j$ we have in the Grothendieck group $K_{0}\left(\mathcal{R}_{2}\right)$

$$
\begin{aligned}
S^{i} \otimes S^{j}= & 2\left(S^{i+j-1}+B e r S^{i+j-3}+\cdots+B e r^{j-1} S^{i-j+1}\right) \\
& +S^{i+j}\left(1+B e r^{-1}\right)+S^{i+j-2}\left(1+B e r^{-1}\right)+\cdots+B e r^{j} S^{i-j}\left(1+B e r^{-1}\right) .
\end{aligned}
$$

For $i=j$ we get

$$
\begin{aligned}
S^{i} \otimes S^{i}= & 2\left(S^{2 i-1}+\operatorname{Ber}^{2 i-3}+\cdots+\operatorname{Ber}^{i-1} S^{1}\right) \\
& +S^{2 i}\left(1+\operatorname{Ber}^{-1}\right)+\cdots+\operatorname{Ber}^{i}\left(1+\operatorname{Ber}^{-1}\right)+B^{i-1}+B^{i-2} .
\end{aligned}
$$

Proof We first consider the cases $S^{i} \otimes S^{1}$ and $S^{i} \otimes S^{2}$ for $i>1$ respectively $i>2$. The case $S^{i} \otimes S^{1}, i>1$ : For the induction start $i=2$ see above. Put $C_{i}=S^{i} \otimes S^{1}$ in $K_{0}\left(\mathcal{R}_{n}\right)$. For $i \geq$ 4 we get then the uniform formula $S^{i} \otimes S^{1}+2 S^{i-1} \otimes S^{1}+S^{i-2} \otimes S^{1}=\left(S^{i+1}+2 S^{i}+S^{i-1}\right)+$ $\left(S^{i-1}+2 S^{i-2}+S^{i-3}\right)+\left(2 C_{i-1}+B e r^{-1} S^{i+1}+B_{e r}{ }^{-1} S^{i-1}+B e r S^{i-1}+B e r S^{i-3}\right)$. Hence using the induction assumption $S^{i-2} \otimes S^{1}=2 S^{i-2}+S^{i-1}+B e r^{-1} S^{i-1}+S^{i-3}+B e r S^{i-3}$ we get $S^{i} \otimes S^{1}=2 S^{i}+S^{i+1}+S^{i-1}+B e r^{-1} S^{i+1}+B e r S^{i-1}$, and this proves the induction step. Likewise for $S^{i} \otimes S^{2}$. Now assume $i>j>2$. Then for $\mathbb{A}_{S^{i}} \otimes \mathbb{A}_{S^{j}}$ we get using Lemma 4.1 the regular formula in $K_{0}\left(\mathcal{R}_{2}\right)$

$$
\begin{aligned}
\mathbb{A}_{S^{i}} \otimes \mathbb{A}_{S^{j}}= & S^{i} \otimes S^{j}+4\left(S^{i-1} \otimes S^{j-1}\right)+2\left(S^{i-1} \otimes S^{j}\right) \\
& +2\left(S^{i-1} \otimes S^{j-2}\right)+2\left(S^{i} \otimes S^{j-1}\right)+S^{i} \otimes S^{j-2} \\
& +2\left(S^{i-2} \otimes S^{j-1}\right)+S^{i-2} \otimes S^{j}+S^{i-2} \otimes S^{j-2} .
\end{aligned}
$$

All tensor products except $S^{i} \otimes S^{j}$ are known by induction. On the other hand this sum equals $\mathbb{A}_{S^{i+j}}+2 \mathbb{A}_{S^{i+j}}+\mathbb{A}_{S^{i+j-2}}+P[i+j-2,0]+2 P[i+j-3,0]+P[i+j-4,0](1+$ $B)+2 B P[i+j-5,0]+B P[i+j-6,0](1+B)+\ldots+2 B^{j-2} P[i-j+1,0]+$ $B^{j-2} P[i-j, 0](1+B)$. Plugging in $P[a, 0]=2 \mathbb{A}_{S^{a+1}}+B^{-1} \mathbb{A}_{S^{a+2}}+\mathbb{A}_{S^{a}}$ for all $a \geq 1$ and comparing terms with the same $B$-power on both sides finishes the proof. The case $i=j$ works exactly the same way.

\subsection{The $\mathcal{R}_{2}$-Case: Socle Estimates}

We say $w(M)=k$ for a module $M$ if $M^{\vee} \cong \operatorname{Ber}^{-k} M$. Examples: $w\left(S^{i}\right)=i-1$ and $w(B e r)=2$, and therefore

$$
w\left(S^{i} \otimes S^{j}\right)=i+j-2 .
$$

On the other hand for $*$-selfdual modules $M$ we have

$$
\operatorname{soc}(M) \cong \operatorname{cosoc}(M),
$$


since $*$-duality is trivial on semisimple modules. On the other hand $w(M)=k$ implies $\operatorname{soc}(M)^{\vee} \cong \operatorname{Ber}^{-k} \operatorname{cosoc}(M)$, so that both conditions together imply $w(\operatorname{soc}(M))=k$. Hence being semi-simple, it is a direct sum of modules

$$
\operatorname{soc}(M) \cong \operatorname{soc}^{\prime}(M) \oplus \bigoplus_{v \in \mathbf{Z}} m(v) \cdot \operatorname{Ber}^{v} S^{k+1-2 v}
$$

with $S^{i}=0$ for $i<0$ and certain multiplicities $m(v)$, plus a sum $\operatorname{soc}^{\prime}(M)$ of modules of type

$$
\left(B e r^{v} \oplus B e r^{k-v-j+1}\right) S^{j}
$$

for certain $v \in \mathbf{Z}$ and certain natural numbers $j$ with $k-v-j+1 \neq v$.

Proposition 5.3 For $n \geq 2$ and for $i>j \geq 2$ we have $\operatorname{soc}^{\prime}(M)=0$ for $M=S^{i-1} \otimes S^{j-1}$ and in $K_{0}\left(\mathcal{R}_{n}\right)$

$$
\operatorname{soc}\left(S^{i-1} \otimes S^{j-1}\right) \hookrightarrow 3 \cdot S^{i+j-3}+2 \cdot B e r S^{i+j-5}+\cdots+2 \cdot B e r^{j-2} S^{i-j+1} .
$$

For $i=j \geq 2$ we have in $K_{0}\left(\mathcal{R}_{n}\right)$

$$
\operatorname{soc}\left(S^{i-1} \otimes S^{i-1}\right) \hookrightarrow 3 \cdot S^{2 i-3}+2 \cdot B e r S^{2 i-5}+\cdots+2 \cdot \operatorname{Ber}^{i-2} S^{1}+B^{i-4} .
$$

Proof Assume $i>j$. Note that $\operatorname{soc}(M) \hookrightarrow \operatorname{soc}\left(\mathbb{A}_{S^{i}} \otimes \mathbb{A}_{S^{j}}\right)$ and by the formula (1) from above the latter is (using $\operatorname{soc}(P[a, b])=[a, b]=\operatorname{Ber}^{b} S^{a-b}$ )

$$
\begin{aligned}
S^{i+j-1} & +3 S^{i+j-2}+3 S^{i+j-3}+(B e r+\mathbf{1}) S^{i+j-4}+2 B e r S^{i+j-5} \\
& +(B e r+\mathbf{1}) B e r S^{i+j-6}+2 B e r^{2} S^{i+j-7}+\cdots \\
& +(B e r+\mathbf{1}) B e r^{j-2} S^{i-j}+2 B e r^{j-2} S^{i-j+1} .
\end{aligned}
$$

Since $k=w(M)=(i-1)-1+(j-1)-1=i+j-4$, this implies the assertion $\operatorname{soc}^{\prime}(M)=0$. Indeed the terms $S^{i+j-1}+3 S^{i+j-2}$ and also $N=(B e r+\mathbf{1}) B^{v e r} S^{i+j-4-2 v}$ cannot contribute to $\operatorname{soc}^{\prime}(M)$, since

$$
\begin{aligned}
N^{\vee} & =\left(\text { Ber }^{-1}+\mathbf{1}\right) \mathrm{Ber}^{-v} \mathrm{Ber}^{-i-j+3+2 v} S^{i+j-4-2 v} \\
& =\left(\mathrm{Ber}^{-1}+\mathbf{1}\right) \mathrm{Ber}^{-i-j+3+v} S^{i+j-4-2 v}
\end{aligned}
$$

and

$$
\begin{aligned}
\mathrm{Ber}^{-k} N & =\mathrm{Ber}^{-k}(\mathrm{Ber}+\mathbf{1}) \mathrm{Ber}^{v} S^{i+j-4-2 v} \\
& =\left(\mathrm{Ber}^{2}+\mathrm{Ber}\right) \mathrm{Ber}^{-i-j+3+v} S^{i+j-4-2 v}
\end{aligned}
$$

have no common irreducible summand. Hence $\operatorname{soc}(M)$ is contained in $3 \cdot S^{i+j-3}+2$. $B e r S^{i+j-5}+\cdots+2 \cdot B e r^{j-2} S^{i-j+1}$. The proof is analogous for $i=j$.

\subsection{The Duflo-Serganova Functor DS}

We recall some constructions from the article [14].

An embedding. We view $G_{n-1}=G L(n-1 \mid n-1)$ as an 'outer block matrix' in $G_{n}=$ $G L(n \mid n)$ and $G_{1}$ as the 'inner block matrix' at the matrix positions $n \leq i, j \leq n+1$. Fix the following element $x \in \mathfrak{g}_{1}$,

$$
x=\left(\begin{array}{ll}
0 & y \\
0 & 0
\end{array}\right) \text { for } y=\left(\begin{array}{cccc}
0 & 0 & \ldots & 0 \\
0 & 0 & \ldots & 0 \\
\ldots & \ldots & \\
1 & 0 & 0 & 0
\end{array}\right) \text {. }
$$


We furthermore fix the embedding

$$
\varphi_{n, 1}: G_{n-1} \times G_{1} \hookrightarrow G_{n}
$$

defined by

$$
\left(\begin{array}{ll}
A & B \\
C & D
\end{array}\right) \times\left(\begin{array}{ll}
a & b \\
c & d
\end{array}\right) \mapsto\left(\begin{array}{llll}
A & 0 & 0 & B \\
0 & a & b & 0 \\
0 & c & d & 0 \\
C & 0 & 0 & D
\end{array}\right) .
$$

We use this embedding to identify elements in $G_{n-1}$ and $G_{1}$ with elements in $G_{n}$. In this sense $\epsilon_{n}=\epsilon_{n-1} \epsilon_{1}$ holds in $G_{n}$ (i.e. $\left.\varphi_{n, 1}\left(\epsilon_{n-1}, \epsilon_{1}\right)=\epsilon_{n}\right)$, for the corresponding elements $\epsilon_{n-1}$ and $\epsilon_{1}$ in $G_{n-1}$ resp. $G_{1}$, defined in Section 2.

Two functors. One has a functor $(V, \rho) \mapsto V^{+}=\left\{v \in V \mid \rho\left(\epsilon_{1}\right)(v)=v\right\}$

$$
+: \mathcal{R}_{n} \rightarrow \mathcal{R}_{n-1}
$$

where $V^{+}$is considered as a $G_{n-1}$-module using $\rho\left(\epsilon_{1}\right) \rho(g)=\rho(g) \rho\left(\epsilon_{1}\right)$ Similarly define $V^{-}=\left\{v \in V \mid \rho\left(\epsilon_{1}\right)(v)=-v\right\}$. With the grading induced from $V=V_{0} \oplus V_{1}$ this defines a representation $V^{-}$of $G_{n-1}$ in $\Pi \mathcal{R}_{n-1}$. Obviously

$$
\left.(V, \rho)\right|_{G_{n-1}}=V^{+} \oplus V^{-} .
$$

Cohomological tensor functors. Since $x$ is an odd element with $[x, x]=0$, we get

$$
2 \cdot \rho(x)^{2}=[\rho(x), \rho(x)]=\rho([x, x])=0
$$

for any representation $(V, \rho)$ of $G_{n}$ in $\mathcal{R}_{n}$. Notice $d=\rho(x)$ supercommutes with $\rho\left(G_{n-1}\right)$. Furthermore $\rho(x): V^{ \pm} \rightarrow V^{\mp}$ holds as a $k$-linear map, an immediate consequence of $d \rho\left(\varepsilon_{1}\right)=-\rho\left(\varepsilon_{1}\right) d$, i.e. of $A d\left(\varepsilon_{1}\right)(x)=-x$. Since $\rho(x)$ is an odd morphism, $\rho(x)$ induces the following even morphisms (morphisms in $\mathcal{R}_{n-1}$ )

$$
\rho(x): V^{+} \rightarrow \Pi\left(V^{-}\right) \quad \text { and } \quad \rho(x): \Pi\left(V^{-}\right) \rightarrow V^{+} .
$$

The $k$-linear map $\partial=\rho(x): V \rightarrow V$ is a differential and commutes with the action of $G_{n-1}$ on $(V, \rho)$. Therefore $\partial$ defines a complex in $\mathcal{R}_{n-1}$

$$
\partial \stackrel{-}{\longrightarrow} V^{+} \partial \stackrel{-}{\longrightarrow} \Pi\left(V^{-}\right) \partial \stackrel{-}{\longrightarrow} V^{+} \partial \stackrel{-}{\longrightarrow} \cdots
$$

Since this complex is periodic, it has essentially only two cohomology groups denoted $H^{+}(V, \rho)$ and $H^{-}(V, \rho)$ in the following. This defines two functors $(V, \rho) \mapsto$ $D_{n, n-1}^{ \pm}(V, \rho)=H^{ \pm}(V, \rho)$

$$
D_{n, n-1}^{ \pm}: \mathcal{R}_{n} \rightarrow \mathcal{R}_{n-1} .
$$

For the categories $\mathcal{T}=\mathcal{T}_{n}$ resp. $\mathcal{T}_{n-1}$ (for the groups $G_{n}$ resp. $G_{n-1}$ ) consider the tensor functor of Duflo and Serganova in [10]

$$
D S_{n, n-1}: \mathcal{T}_{n} \rightarrow \mathcal{T}_{n-1}
$$

defined by $D S_{n, n-1}(V, \rho)=V_{x}:=\operatorname{Kern}(\rho(x)) / \operatorname{Im}(\rho(x))$. Then for $(V, \rho) \in \mathcal{R}_{n}$

$$
H^{+}(V, \rho) \oplus \Pi\left(H^{-}(V, \rho)\right)=D S_{n, n-1}(V) .
$$

Indeed, the left side is $D S_{n, n-1}(V)=V_{x}$ for the $k$-linear map $\partial=\rho(x)$ on $V=$ $V^{+} \oplus V^{-}$. Hence $H^{+}$is the functor obtained by composing the tensor functor

$$
D S_{n, n-1}: \mathcal{R}_{n} \rightarrow \mathcal{T}_{n-1}
$$

with the functor

$$
\mathcal{T}_{n-1} \rightarrow \mathcal{R}_{n-1}
$$

that projects the abelian category $\mathcal{T}_{n-1}$ onto $\mathcal{R}_{n-1}$ using $\mathcal{T}_{n}=\mathcal{R}_{n} \oplus \Pi \mathcal{R}_{n}$. 
The ring homomorphism $d$. As an element of the Grothendieck group $K_{0}\left(\mathcal{R}_{n-1}\right)$ we define for a module $M \in \mathcal{R}_{n}$

$$
d(M)=H^{+}(M)-H^{-}(M) .
$$

The map $d$ is additive by [14]. Notice

$$
K_{0}\left(\mathcal{T}_{n}\right)=K_{0}\left(\mathcal{R}_{n}\right) \oplus K_{0}\left(\mathcal{R}_{n}[1]\right)=K_{0}\left(\mathcal{R}_{n}\right) \otimes(\mathbb{Z} \oplus \mathbb{Z} \cdot \Pi)
$$

We have a commutative diagram

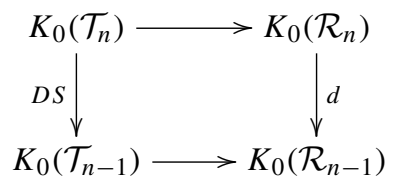

where the horizontal maps are surjective ring homomorphisms defined by $\Pi \mapsto-1$. Since $D S$ induces a ring homomorphism, $d$ defines a ring homomorphism.

\subsection{The $\mathcal{R}_{2}$-Case: Indecomposability}

If we display the maximal atypical composition factors $[x, y]$ of $S^{i} \otimes S^{j}$ in the $\mathbf{Z}^{2}$-lattice, we get the following picture. Here $\square$ denotes composition factors occuring with multiplicity 2 and the $\circ$ appear with multiplicity 1 . The socle is contained in the subset of composition factors denoted by $\square$.

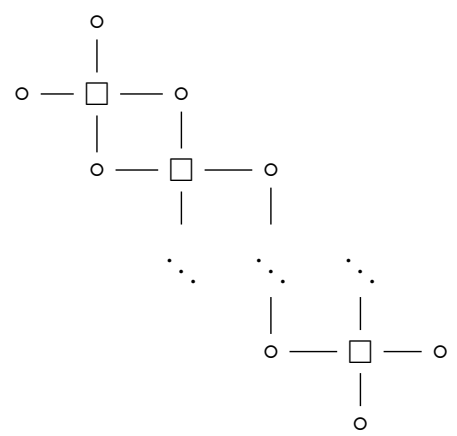

with the two o to the upper left at position $B^{j} S^{i-j}$ and $B^{j-1} S^{i-j}$ and the ones to the lower right at position $B^{-1} S^{i+j}$ and $S^{i+j}$. The picture in the $i=j$-case is similar

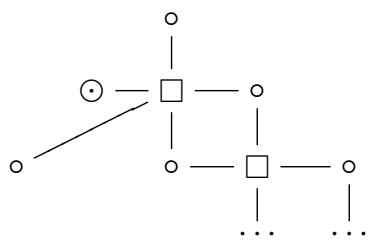

with the composition factor $\odot$ at position $B^{i-1}$ appearing with multiplicity 2 and the additional $\circ$ at position $B^{i-2}$. 
We now make use of the cohomological tensor functors $D S$. In the $G L(1 \mid 1)$-case $S^{i} \simeq$ $B^{i}$ and hence $S^{i} \otimes S^{j}=S^{i+j}$. We know from [14] that $D S\left(S^{i}\right)=S^{i}+\Pi^{1-i} B^{-1}$ and $D S(B)=\Pi^{-1} B$. Hence $D S\left(S^{i} \otimes S^{j}\right)$ splits into four indecomposable summands each of superdimension 1 or each of superdimension -1 :

$$
\begin{aligned}
D S\left(S^{i} \otimes S^{j}\right) & =\left(S^{i} \oplus \Pi^{1-i} B^{-1}\right) \otimes\left(S^{j} \oplus \Pi^{1-j} B^{-1}\right) \\
& =B^{i+j} \oplus \Pi^{1-j} B^{i-1} \oplus \Pi^{1-i} B^{j-1} \oplus \Pi^{2-i-j} B^{-2} .
\end{aligned}
$$

Hence $M=S^{i} \otimes S^{j}$ splits into at most four indecomposable summands of $s d i m \neq 0$.

Lemma 5.4 Every atypical direct summand is *-invariant.

Proof If $I$ is a direct summand which is not *-invariant, $M$ contains $I^{*}$ as a direct summand and $[I]=\left[I^{*}\right]$ in $K_{0}\left(\mathcal{R}_{n}\right)$ since ${ }^{*}$ identity on irreducible modules. However any summand of length $>1$ must contain a factor of type $\circ$ which occur in $M$ only with multiplicity 1 , a contradiction.

Corollary 5.5 The superdimension of any maximally atypical summand is $\neq 0$.

Proof $M$ does not contain any projectice cover (look at composition factors). If $\operatorname{sim}(I)=$ $0, D S(I)=0$. However $\operatorname{ker}(D S)=\mathcal{C}^{-}[14$, Theorem 4.1] (the modules with a filtration by anti-Kac-modules) which are not $*$-invariant, unless they are projective.

Assume $i>j$. By $*$-invariance the Loewy length of a direct summand is either 1 or 3 . If $I$ is irreducible, then necessarily $I=\square$ for a composition factor of the socle. By socle considerations both $\square$ will split as direct summands. The remaining module would have superdimension zero, hence the Loewy length of a direct summand is 3. Fix a composition factor of type $\square$. The multiplicity of $\square$ in the socle cannot be 2. If the multiplicity of $\square$ in the socle is zero, then $\square$ has to be in the middle Loewy layer. But this would force composition factors of type o to be in the socle. Contradiction. Hence

Corollary 5.6 For $n \geq 2$ and $i>j$

$$
\operatorname{soc}\left(S^{i} \otimes S^{j}\right)=S^{i+j-1} \oplus \operatorname{Ber} S^{i+j-3} \oplus \cdots \oplus \operatorname{Ber}^{j-1} S^{i-j+1} .
$$

We conclude that the superdimension of a direct summand is either 2 or 4 . Hence $M$ is either indecomposable or splits into two summands $M=I_{1} \bigoplus I_{2}$ of superdimension 2. If $M$ would split, it would split in the following way:
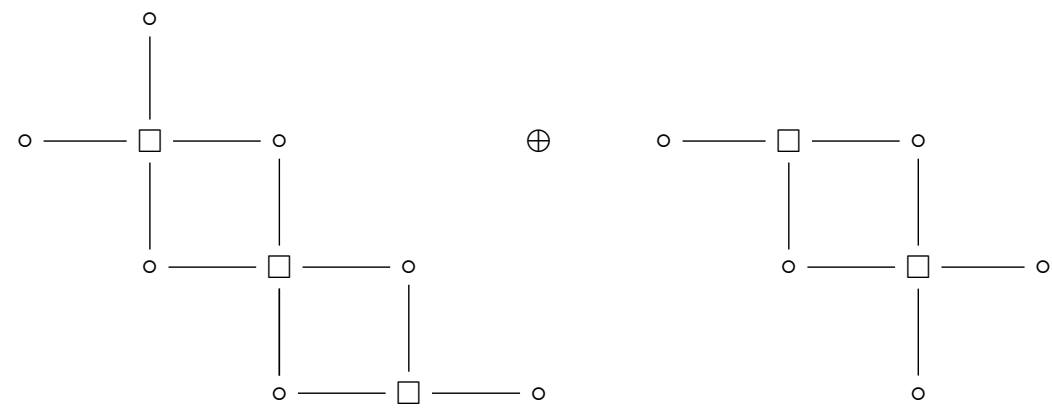
Now we use the ring homomorphism $d: K_{0}\left(\mathcal{R}_{n}\right) \rightarrow K_{0}\left(\mathcal{R}_{n-1}\right)$ defined by $d(M)=$ $H^{+}(M)-H^{-}(M)$ as above. We know

$$
d\left(S^{i} \otimes S^{j}\right)=B^{i+j}+(-1)^{1-j} B^{i-1}+(-1)^{1-i} B^{j-1}+(-1)^{2-i-j} B^{-2}
$$

since we can just take the formula for $D S\left(S^{i} \otimes S^{j}\right)$ and replace the parity shifts $\Pi^{i}$ by $(-1)^{i}$. Since $D S$ maps Anti-Kac modules to zero, $d$ applied to any square with edges $B^{k} S^{i}$, $B^{k+1} S^{i-1}, B^{k+1} S^{i}, B^{k} S^{i+1}$ is zero. Hence $d\left(I_{2}\right)$ is given by applying $d$ to the hook in the lower right $d\left(S^{i+j}+S^{i+j-1}+B^{-1} S^{i+j}\right)$ and to $\left(B^{v} S^{i+j+1-2 v}+B^{v} S^{i+j-2 v}\right)$ from the upper left of $I_{2}$. We get $d\left(I_{2}\right)=B^{i+j}+(-1)^{i-j} B^{-2}+(-1)^{v} B^{i+j+1-v}+(-1)^{v} B^{i+j-v}$ with the two additional summands $(-1)^{v} B^{i+j+1-v}+(-1)^{v} B^{i+j-v}$. Contradiction, hence $M$ is indecomposable.

Now assume $i=j$. By the socle estimates for $S^{i} \otimes S^{i}$ and $*$-duality either $B^{i-1}$ splits as a direct summand or both $B^{i-1}$ lie in the middle Loewy layer. Note that $\operatorname{Hom}\left(B^{i-1}, S^{i} \otimes\right.$ $\left.S^{i}\right)=\operatorname{Hom}\left(B^{i-1} \otimes\left(S^{i}\right)^{\vee}, S^{i}\right)=\operatorname{End}\left(S^{i}\right)=k$, hence the last case cannot happen. Hence $B^{i-1}$ splits as a direct summand. We show that the remaining module $M^{\prime}$ in $S^{i} \otimes S^{i}=$ $B^{i-1} \oplus M^{\prime}$ is indecomposable. As in the $i>j$-case the Loewy length of any direct summand of $M^{\prime}$ must be 3 . As before we obtain for $i=j$

$$
\operatorname{soc}\left(S^{i} \otimes S^{i}\right)=S^{2 i-1} \oplus \operatorname{Ber}^{2 i-3} \oplus \cdots \oplus \operatorname{Ber}^{i-1} S^{1} \oplus B^{i-1} .
$$

The remaining part $M^{\prime}$ can either split into three indecomposable modules of superdimension one each, in a direct sum of two modules of superdimension one respectively two or is indecomposable. One cannot split the upper left $\tilde{I}$

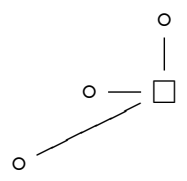

as a direct summand since its superdimension is -1 . Similarly one cannot split

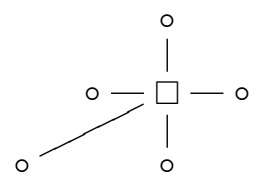

as a direct summand since the remaining module would have superdimension zero. Since all composition factors except the $B$ 's have superdimension $\pm 2, M^{\prime}$ could split only into $M^{\prime}=I_{1} \oplus I_{2}$ with $\operatorname{sim}\left(I_{1}\right)=1$ and $\operatorname{sim}\left(I_{2}\right)=2$ with $I_{2}$ as above. We argue now as in the $i>j$-case. In the Grothendieck ring $K_{0}\left(\mathcal{R}_{n}\right)$

$$
d(M)=B^{2 i}+(-1)^{1-i} B^{i-1}+(-1)^{1-i} B^{i-1},
$$

but $d\left(I_{2}\right)$ has four summands as in the $i>j$-case. Contradiction, hence $M$ is indecomposable.

Corollary 5.7 Up to summands which are not in the maximal atypical block we obtain $S^{i} \otimes S^{j} \simeq M(i>j)$ where $M$ is indecomposable with Loewy structure

$$
\left(\begin{array}{llll}
S^{i+j-1} & \operatorname{Ber}^{i+j-3} & \ldots & \operatorname{Ber}^{j-1} S^{i-j+1} \\
S^{i+j}\left(1+\operatorname{Ber}^{-1}\right) & \ldots & \operatorname{Ber}^{j} S^{i-j}\left(1+B e r^{-1}\right) \\
S^{i+j-1} & \operatorname{Ber}^{i+j-3} & \ldots & \operatorname{Ber}^{j-1} S^{i-j+1}
\end{array}\right)
$$


and $S^{i} \otimes S^{i}=B^{i-1} \oplus M$ where $M$ is indecomposable with Loewy structure

$$
\left(\begin{array}{llcl}
S^{2 i-1} \quad \operatorname{Ber}^{2 i-3} & \ldots & \operatorname{Ber}^{i-1} S^{1} & \\
S^{2 i}\left(1+\operatorname{Ber}^{-1}\right) & \cdots & \operatorname{Ber}^{i} S^{0}\left(1+\operatorname{Ber}^{-1}\right) & B^{i-2} \\
S^{2 i-1}+\operatorname{Ber}^{2 i-3} & \cdots & \operatorname{Ber}^{i-1} S^{1}
\end{array}\right) .
$$

We remark that the summand $B e r^{i-1}$ in $\left(\Pi^{i} S^{i}\right)^{\otimes 2}$ belongs to $\Lambda^{2}\left(\Pi^{i} S^{i}\right)$ and the summand $M$ to $\operatorname{Sym}^{2}\left(\Pi^{i} S^{i}\right)$, see also [15]. Note that $\Lambda^{2}(\Pi(V))=\operatorname{Sym}^{2}(V)$ for $V \in$ $\mathcal{R}_{n}$.

\subsection{The GL(2|2)-Case}

It is worth summarizing the situation in the $n=2$-case. In the $G L(2 \mid 2)$-case the irreducible representations are either typical, singly atypical or double (maximal) atypical. Every typical representation is a mixed tensor and every singly atypical irreducible representation is a Berezin twist of a mixed tensor. Hence the results of [13] give the decomposition law for tensor products between typical and/or singly atypical irreducible representations. In [13, Remark 13.4] it is also explained how to decompose the tensor products between a typical and an irreducible maximal atypical representation in the $G L(2 \mid 2)$-case. Hence the fusion rules between irreducible representations are known except for the tensor product of a singly atypical and a maximal atypical representation, but these could be calculated by imitating our approach in the maximal atypical case. Since every irreducible maximal atypical representation of $G L(2 \mid 2)$ is of the form $[a, b]$ and any such representation is a Berezin twist of one of the $S^{i}=[i, 0]$ for the Berezin determinant $B e r$, our result covers the entire maximal atypical $G L(2 \mid 2)$-case. For the $\mathfrak{p s l}(2 \mid 2)$-case these decompositions were found prior by physicists [12].

\section{Reduction to the $G L(2 \mid 2)$-Case}

In this section we show that the determination of the maximal atypical summands in $S^{i} \otimes S^{j}$ in $\mathcal{R}_{n}$ is a corollary of the $n=2$-case if we use the formalism of [15]. Therefore this section depends unlike the other sections on some results of [15].

\subsection{Clean Decomposition}

We do not calculate the maximal atypical composition factors of $S^{i} \otimes S^{j}$ for $n \geq 3$. Nonetheless we can determine the number of indecomposable summands and their superdimension. We assume $n \geq 2$ and $i \geq j$.

Lemma 6.1 The Loewy length of a direct summand in $S^{i} \otimes S^{j}$ or $S^{i} \otimes\left(S^{j}\right)^{\vee}$ is $\leq 5$.

Proof Since $S^{i}$ is in the socle and top of $\mathbb{A}_{S^{i+1}}$ we have a surjection $\mathbb{A}_{S^{i+1}} \otimes \mathbb{A}_{S^{j+1}} \rightarrow$ $S^{i} \otimes S^{j}$. By the explicit formulas for $\mathbb{A}_{S^{i+1}} \otimes \mathbb{A}_{S^{j+1}}$, the maximal Loewy length of a summand in $\mathbb{A}_{S^{i+1}} \otimes \mathbb{A}_{S^{j+1}}$ is $\leq 5$. For that recall that the Loewy length of a mixed tensor $R(\lambda)$ equals $2 d(\lambda)+1$, and it is easy to check that $(a, b)$ satisfies $d(a, b)=2$. Hence the quotient $S^{i} \otimes S^{j}$ has Loewy length at most 5 . The case $S^{i} \otimes\left(S^{j}\right)^{\vee}$ is proved in the same manner. 
Since the Loewy length of a maximal atypical projective cover in $\mathcal{R}_{n}$ is $2 n+1$ by [4, Theorem 5.1] we get

Corollary 6.2 For all $n$ no maximal atypical projective cover appears in the decompositions $S^{i} \otimes S^{j}$ resp. $S^{i} \otimes\left(S^{j}\right)^{\vee}$.

Proof For $n=2$ we saw this by brute force computations. For $n \geq 3$ we have $2 n+1>$ 5 .

We say a direct sum is clean if none of the summands is negligible (i.e. has superdimension 0 ). We say a negligible module in $\mathcal{R}_{n}$ is potentially projective of degree $r$ if $D S^{n-r}(N) \in \mathcal{T}_{r}$ is projective and $D S^{i}(N)$ is not for $i \leq n-r$.

Lemma 6.3 Every maximal atypical negligible summand in a tensor product $L(\lambda) \otimes L(\mu)$ is potentially projective of degree at least 3.

We proved in [15, Corollary 5.8] that the kernel of $D S$ equals Proj if we restrict $D S$ to the full subcategory $\mathcal{T}_{n}^{ \pm}$of indecomposable modules occuring as direct summands in an iterated tensor product of irreducible modules.

Proof The maximal atypical part of the decomposition of $S^{i} \otimes S^{j}$ in $\mathcal{R}_{2}$ is clean by Corollary 5.7. Further $D S$ sends negligible modules in $\mathcal{T}_{n}^{ \pm}$to negligible modules in $\mathcal{T}_{n-1}^{ \pm}$ [15, Ccorollary 5.5] and the kernel of $D S$ on $\mathcal{T}_{n}^{ \pm}$consists of the projective elements. Since $D S^{n-2}(L(\lambda) \otimes L(\mu)) \in \mathcal{T}_{2}$ splits into a direct sum of irreducible representations of the form $B^{a} S^{b}$ for some $a, b \in \mathbf{Z}$ by our $G L(2 \mid 2)$-computations, $D S^{n-2}(N)=0$.

Lemma 6.4 For all $n$ the projection of $S^{i} \otimes S^{j}$ or $S^{i} \otimes\left(S^{j}\right)^{\vee}$ on the maximal atypical block is clean.

Proof We know that this is true for $n=2$. If $N$ is a maximal atypical summand in $S^{i} \otimes S^{j}$, we apply $D S$ several times until $N$ becomes projective. Since $D S\left(S^{i}\right)=S^{i}$ for $i<n-1$ and $D S\left(S^{i}\right)=S^{i} \oplus \Pi^{n-1-i} B_{e r}^{-1}$ for $i \geq n-1$, the tensor product $D S \circ \ldots \circ D S\left(S^{i} \otimes S^{j}\right)$ splits into a tensor product of $S^{i}$,s and Berezin powers. The projective summand coming from $N$ gives now a contradiction to Corollary 6.2. In the $S^{i} \otimes\left(S^{j}\right)^{\vee}$-case we can argue in the same way using $D S\left(\left(S^{j}\right)^{\vee}\right)=D S\left(S^{j}\right)^{\vee}$.

\subsection{Nonvanishing Superdimension}

In this part we refer extensively to results from [15]. We conclude from the previous paragraph that all maximal atypical summands in $S^{i} \otimes S^{j}$ have non-vanishing superdimension. Hence the direct summands can be seen in the quotient $\mathcal{R}_{n} / \mathcal{N}$ by the modules of superdimension 0 . According to $[15,16]$ the tensor subcategory generated by the image of an irreducible element $L$ in this quotient is of the form $\operatorname{Rep}\left(H_{L}, \epsilon\right)$ for some algebraic supergroup $H_{L}$ and some twist $\epsilon$ as in Section 2. We apply this to the representations $S^{i}$. By abuse of notation we denote the image of $S^{i}$ in the quotient still by $S^{i}$. We show in [15] that the connected derived group $\left(H_{S^{i}}^{0}\right)_{d e r}$ of $S^{i}$ always satisfies

$$
\left(H_{S^{i}}^{0}\right)_{d e r} \simeq S L(i+1) \text { for } i \leq n-2
$$


and

$$
\left(H_{S^{i}}^{0}\right)_{d e r} \simeq S L(n) \text { for } i \geq n-1 .
$$

Furthermore the restriction of $S^{i}$ (seen as a representation of $\left.H_{S^{i}}\right)$ to $\left(H_{S^{i}}\right)_{d e r}^{0}$ remains irreducible. By superdimension reasons this restriction corresponds to the standard representation of $S L(i+1)$ or $S L(n)$ respectively. The derived group of the group corresponding to the tensor category $<S^{i}, S^{j}>, j \neq i$ generated by $S^{i}$ and $S^{j}$ in the quotient is the direct product of the derived groups corresponding to $S^{i}$ and $S^{j}$. If $S^{i} \otimes S^{j}$ would decompose as $M_{1} \oplus M_{2}$ (up to superdimension 0), the restriction to the derived group

$$
H_{d e r}^{0} \simeq\left(H_{S^{i}}^{0}\right)_{d e r} \times\left(H_{S^{j}}^{0}\right)_{d e r}
$$

would give a decomposition $\operatorname{Res}\left(M_{1}\right) \oplus \operatorname{Res}\left(M_{2}\right)$ of the tensor product $\operatorname{Res}\left(S^{i}\right) \otimes \operatorname{Res}\left(S^{j}\right)$. But this tensor product is the external tensor product of the standard representation of the first factor with the standard representation of the second factor. Hence $S^{i} \otimes S^{j}$ is indecomposable for $i \neq j$ up to superdimension 0 . If $i=j$ then the tensor product $S^{i} \otimes S^{i}$ behaves up to summands of superdimension 0 like the tensor product of the standard representation of $S L(n)$ with itself. Since this tensor product splits into the two irreducible representations of weight $(2,0, \ldots, 0)$ and $(1,1,0, \ldots, 0), S^{i} \otimes S^{i}$ has two indecomposable summands of non-vanishing superdimension.

Corollary 6.5 The tensor product $S^{i} \otimes S^{j}$ in $\mathcal{R}_{n}$ has a single indecomposable maximal atypical summand for $i \neq j$ and decomposes in two indecomposable summands for $i=j$.

Remark 6.6 In other words, once the know the $G L(2 \mid 2)$-case we get Corollary 6.5 for free using the formalism of $[14,15]$. Note that we need very little of the machinery in [15] in the $S^{i}$-case since this can be treated in an adhoc way [15, Section 9].

\section{The Lower Atypical Summands in $\mathcal{R}_{n}$}

We compute the remaining direct summands of the tensor product $S^{i} \otimes S^{j}$ in $\mathcal{R}_{n}$ for $n \geq 2$. These direct summands are all irreducible which will follow from the fact that all summands of atypicality $<n$ in an $\mathbb{A}_{S^{i}} \otimes \mathbb{A}_{S^{j}}$ tensor product are irreducible and have vanishing $E x t^{1}$ with each other.

Lemma 7.1 $\mathbb{A}_{S^{i}} \otimes \mathbb{A}_{S^{j}}$ is a direct sum of maximally atypical summands and irreducible representations of atypicality $n-2$ and likewise for $\mathbb{A}_{\Lambda^{i}} \otimes \mathbb{A}_{\Lambda^{j}}$.

Proof In the decomposition of $\operatorname{lift}\left(\left(i ; 1^{i}\right) \otimes\left(j ; 1^{j}\right)\right)$ in $R_{t}$, the bipartitions which will not contribute to the maximal atypical block are by the formulas in Section 4.3 of the form

$$
\left((i+j-k, k) ;\left(2^{r}, 1^{i+j-2 r}\right)\right)
$$

for some $k, r \geq 0$ and $k \neq r$ (note that the $k=r$ part would include the maximal atypical contributions that we already calculated). We have

$$
\begin{aligned}
& I_{\wedge}=\{i+j-k, k-1,-2,-3,-4, \ldots\} \\
& I_{\vee}=\{-1,0,1, \ldots, r-2, r, r+1, \ldots, i+j-r-1, i+j-r+1, \ldots\}
\end{aligned}
$$

Since $k \neq r$, neither one of the two conditions $i+j-k=i+j-r, k-1=r-1$ is satisfied, hence the two sets intersect at two points, hence the weight diagram of any such 
bipartition has two crosses and two circles. Clearly the weight diagrams do not have any $\vee \wedge$-pair, hence the corresponding modules are irreducible.

Lemma 7.2 The composition factors of $S^{i} \otimes S^{j}$ in $\mathcal{R}_{n}$ which are not maximally atypical are given by the set

$$
R\left((i+j-k, k) ;\left(2^{r}, 1^{i+j-2 r}\right)\right), k, r=0,1, \ldots, \min (i, j), k \neq r .
$$

All these modules have atypicality $n-2$ and are irreducible.

Proof This is again a recursive determination from the $\mathbb{A}_{S^{i}} \otimes \mathbb{A}_{S^{j}}$ tensor products. As before the $S^{i} \otimes S^{1}$ and $S^{i} \otimes S^{2}$-cases for $i \geq 1$ respectively $i \geq 2$ should be treated separately. For $S^{i} \otimes S^{j}, i, j \geq 3$ we obtain the regular formulas

$$
\begin{aligned}
\mathbb{A}_{S^{i}} \otimes \mathbb{A}_{S^{j}} & =\left(S^{i}+2 S^{i-1}+S^{i-2}\right) \otimes\left(S^{j}+2 S^{j-1}+S^{j-2}\right) \\
& =S^{i} \otimes S^{j}+\text { lower terms }
\end{aligned}
$$

where the lower terms are known by induction. Recall from Section 4.2.2 that $\Gamma_{\lambda \mu}^{v}=0$ unless $|v| \leq\left(r+r^{\prime}, s+s^{\prime}\right)$. In the $\mathbb{A}_{S^{i}} \otimes \mathbb{A}_{S^{j}}$ tensor product the $R($,$) 's from above can$ therefore not occur for degree reasons in any tensor product $\mathbb{A}_{S^{p}} \otimes \mathbb{A}_{S^{q}}$ for $p \leq i, q \leq j$ where either $p<i$ or $q<j$. Hence they cannot occur in any tensor product decomposition of any $S^{p} \otimes S^{q}$ for $p, q$ as above, hence they have to occur in the $S^{i} \otimes S^{j}$-decomposition. The number of these modules is $\min (i, j)^{2}-\min (i, j)$. Substracting the inductively known numbers of not maximally atypical contributions in $S^{p} \otimes S^{q}$ in the $\mathbb{A}_{S^{i}} \otimes \mathbb{A}_{S^{j}}$-tensor product from the number of all such contributions in $\mathbb{A}_{S^{i}} \otimes \mathbb{A}_{S^{j}}$ we get $\min (i, j)^{2}-\min (i, j)$ remaining modules. Hence there are no other summands in $S^{i} \otimes S^{j}$.

Lemma 7.3 The irreducible representation $R\left((i+j-k, k) ;\left(2^{r}, 1^{i+j-2 r}\right)\right)$ is isomorphic to

$$
L(i+j-k, k, 0, \ldots, 0 \mid 0, \ldots, 0,-r,-i-j+r) .
$$

Proof . Let $m$ denote the maximal coordinate of a cross or circle in the weight diagram of the bipartition. To obtain the weight diagram of the highest weight we have to switch all labels to the right of this coordinate as well as the first $M-n+2$ labels to its left which are not labelled $\times$ or $\circ$ by the explicit description of $\theta$ in [13, 6.1]. Since we have four symbols $\times$ and $\circ$ this amounts to switching all the labels at positions $\geq-1$ and $<M$ (all of them $\vee$ 's) and the $n-2 \wedge$ 's at positions $-2, \ldots,-n+1$ to $\vee$ 's. The crosses are at the positions $i+j-k, k-1$ and the circles at the positions $i+j-r, r-1$. The result follows.

Lemma 7.4 The lower atypical direct summands of $S^{i} \otimes S^{j}$ in $\mathcal{R}_{n}$ are given by the set

$$
R\left((i+j-k, k) ;\left(2^{r}, 1^{i+j-2 r}\right)\right), k, r=0,1, \ldots, \min (i, j), k \neq r .
$$

Proof For any irreducible mixed tensors $R(\lambda), R(\mu)$ we have

$$
\operatorname{Ext}_{1}(R(\lambda), R(\mu))=0
$$

since every block contains a unique irreducible mixed tensor by [13, Lemma 8.1].

For a maximally atypical weight $\left(\lambda_{1}, \ldots, \lambda_{n} \mid-\lambda_{n}, \ldots,-\lambda_{1}\right)$ denote by

$$
L_{0}\left(\lambda_{1}, \ldots, \lambda_{n}\right) \otimes L_{0}\left(-\lambda_{n}, \ldots,-\lambda_{1}\right)
$$


the underlying irreducible $G L(n) \times G L(n)$-module. Denote by $\pi$ the following additive map from irreducible $G L(n) \times G L(n)$ modules to irreducible $G L(n \mid n)$-modules:

$$
\begin{aligned}
& \pi\left(\left(L_{0}\left(\lambda_{1}, \ldots, \lambda_{n}\right) \otimes L_{0}\left(\mu_{1}, \ldots, \mu_{n}\right)\right)\right. \\
= & \begin{cases}0 & L\left(\lambda_{1}, \ldots, \lambda_{n} \mid \mu_{1}, \ldots, \mu_{n}\right) \in \Gamma \\
L\left(\lambda_{1}, \ldots, \lambda_{n} \mid \mu_{1}, \ldots, \mu_{n}\right) & \text { else. }\end{cases}
\end{aligned}
$$

Corollary 7.5 The not maximally atypical contributions to $S^{i} \otimes S^{j}$ are given by

$$
\pi\left(\left(L_{0}(i, 0, \ldots, 0) \otimes L_{0}(0, \ldots, 0,-i)\right) \otimes\left(L_{0}(j, 0, \ldots, 0) \otimes L_{0}(0, \ldots, 0,-j)\right) .\right.
$$

Corollary 7.6 For $n=2$ the tensor product $S^{i} \otimes S^{j}(i>j)$ decomposes as

$$
\begin{aligned}
& S^{i} \quad \otimes S^{j} \simeq\left(\begin{array}{llll}
S^{i+j-1} & \operatorname{Ber}^{i+j-3} & \ldots & \operatorname{Ber}^{j-1} S^{i-j+1} \\
S^{i+j}\left(1+\operatorname{Ber}^{-1}\right) & \ldots & \operatorname{Ber}^{j} S^{i-j}\left(1+\operatorname{Ber}^{-1}\right) \\
S^{i+j-1} & \operatorname{Ber}^{i+j-3} & \ldots & \operatorname{Ber}^{j-1} S^{i-j+1}
\end{array}\right) \\
& \oplus \pi\left(\left(L_{0}(i, 0, \ldots, 0) \otimes L_{0}(0, \ldots, 0,-i)\right) \otimes\left(L_{0}(j, 0, \ldots, 0) \otimes L_{0}(0, \ldots, 0,-j)\right) .\right.
\end{aligned}
$$

The tensor product $S^{i} \otimes S^{i}$ decomposes as

$$
S^{i} \otimes S^{i} \simeq B^{i-1} \oplus\left(\begin{array}{llll}
S^{2 i-1} & \operatorname{Ber}^{2 i-3} & \cdots & \operatorname{Ber}^{i-1} S^{1} \\
S^{2 i}\left(1+\operatorname{Ber}^{-1}\right) & \cdots & \operatorname{Ber}^{i} S^{0}\left(1+\operatorname{Ber}^{-1}\right) & B^{i-2} \\
S^{2 i-1}+\operatorname{Ber}^{2 i-3} & \cdots & \operatorname{Ber}^{i-1} S^{1}
\end{array}\right)
$$

$\oplus \pi\left(\left(L_{0}(i, 0, \ldots, 0) \otimes L_{0}(0, \ldots, 0,-i)\right) \otimes\left(L_{0}(i, 0, \ldots, 0) \otimes L_{0}(0, \ldots, 0,-i)\right)\right.$.

\section{The $G L(3 \mid 3)$-Case and a Conjecture}

The method applied to compute the $S^{i} \otimes S^{j}$ tensor products in the $G L(2 \mid 2)$-case works in principal for arbitrary $n$. Note that the results on the $\mathbb{A}_{S^{i}} \otimes \mathbb{A}_{S^{j}}$ tensor products are valid for any $n$. Furthermore we determined the part of $S^{i} \otimes S^{j}$ which is not maximal atypical for any $n \geq 2$, hence we restrict here to the maximal atypical part. The obstacle to use the method of the $\mathcal{R}_{2}$-case effectively is that the composition factors of the modules $R(a, b)$ appearing in the $\mathbb{A}_{S^{i}} \otimes \mathbb{A}_{S^{j}}$-case are difficult to compute. Decomposing a few $R(a, b)$ for small $a$ and $b$ in the $n=3$-case and then computing the composition factors of the $S^{i} \otimes S^{j}$ tensor products recursively, we arrive at the following tensor products $\left(\Lambda^{2}=\left(S^{2}\right)^{\vee}\right)$. Here we always project to the maximal atypical block.

$$
\begin{gathered}
S^{1} \otimes S^{1} \simeq \mathbf{1} \oplus\left(\begin{array}{lll}
S^{1} & & \\
S^{2}+ & \left(S^{2}\right)^{\vee} & \mathbf{1} \\
S^{1}
\end{array}\right) \\
S^{2} \otimes S^{1} \simeq\left(\begin{array}{llll}
S^{2} & \\
S^{3} & {[2,1,0]} & S^{1} & B^{-1} \\
S^{2} &
\end{array}\right) \\
S^{3} \otimes S^{1} \simeq\left(\begin{array}{lll}
S^{3} & \\
S^{4} & {[3,1,0]} & S^{2} \\
S^{3} &
\end{array}\right)
\end{gathered}
$$




$$
\begin{aligned}
& S^{2} \otimes S^{2} \simeq[1,1,0] \\
& \oplus\left(\begin{array}{llllllll}
S^{3} & {[2,1,0]} \\
S^{4} & {[3,1,0]} & {[2,2,0]} & \left(S^{2}\right)^{\vee} & {[2,-1,-1]} & {[0,-1,-1]} & S^{2} & \mathbf{1} \\
S^{3} & {[2,1,0]}
\end{array}\right)
\end{aligned}
$$

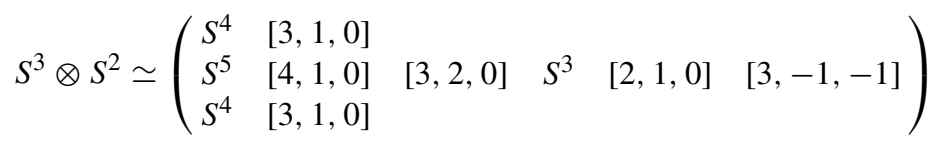

$$
\begin{aligned}
& S^{3} \otimes S^{3} \simeq[2,2,0] \oplus
\end{aligned}
$$

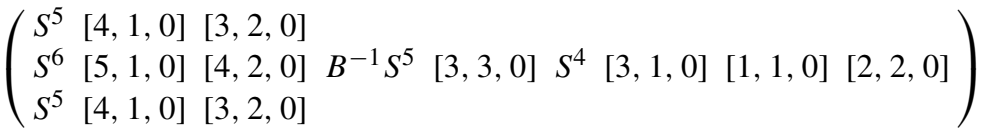

Conjecture 8.1 After projection to the maximal atypical block, $S^{i} \otimes S^{j}=M$ is indecomposable if $i \neq j$ (Corollary 6.5). $S^{i} \otimes S^{i}$ splits as

$$
[i-1, i-1, \ldots, i-1,0] \oplus M .
$$

The socle of $M$ is for $i \geq j$

$$
\operatorname{soc}(M)=[i+j-1,0, \ldots, 0] \oplus[i+j-2,1,0, \ldots, 0] \oplus \ldots \oplus[i, j-1, \ldots, 0]
$$

and $M$ has Loewy length 3.

Note that since $\mathbb{A}_{S^{i}} \rightarrow \mathbb{A}_{S^{j}} \rightarrow S^{i} \otimes S^{j}$ and the maximal Loewy length of a direct summand $R(a, b)$ in $\mathbb{A}_{S^{i}} \rightarrow \mathbb{A}_{S^{j}}$ is 5 , the Loewy length of $M$ is at most 5 .

Funding Information Open Access funding provided by Projekt DEAL.

\section{Compliance with Ethical Standards}

Conflict of Interests On behalf of all authors, the corresponding author states that there is no conflict of interest.

Open Access This article is licensed under a Creative Commons Attribution 4.0 International License, which permits use, sharing, adaptation, distribution and reproduction in any medium or format, as long as you give appropriate credit to the original author(s) and the source, provide a link to the Creative Commons licence, and indicate if changes were made. The images or other third party material in this article are included in the article's Creative Commons licence, unless indicated otherwise in a credit line to the material. If material is not included in the article's Creative Commons licence and your intended use is not permitted by statutory regulation or exceeds the permitted use, you will need to obtain permission directly from the copyright holder. To view a copy of this licence, visit http://creativecommonshorg/licenses/by/4.0/.

\section{References}

1. Berele, A., Regev, A.: Hook Young diagrams with applications to combinatorics and to representations of Lie superalgebras. Adv. Math. 64, 118-175 (1987)

2. Brundan, J.: Kazhdan-Lusztig polynomials and character formulae for the Lie superalgebra $g l(m \mid n)$. J. Am. Math Soc. 16(1), 185-231 (2003) 
3. Brundan, J., Stroppel, C.: Highest weight categories arising from Khovanov's diagram algebra. II: Koszulity, Transform. Groups 15(1), 685-722 (2010)

4. Brundan, J., Stroppel, C.: Highest weight categories arising from Khovanov's diagram algebra. I: cellularity, Mosc. Math. J. 11(4), 170-243 (2011)

5. Brundan, J., Stroppel, C.: Highest weight categories arising from Khovanov's diagram algebra. IV: the general linear supergroup. J. Eur. Math. Soc. (JEMS) 14(2), 373-419 (2012)

6. Brundan, J., Stroppel, C.: Gradings on walled Brauer algebras and Khovanov's arc algebra. Adv. Math. 231(2), 709-773 (2012)

7. Comes, J., Wilson, B.: Deligne's category $\operatorname{Rep}\left(G L_{\delta}\right)$ and representations of general linear supergroups. Represent. Theory 16, 568-609 (2012)

8. Deligne, P.: La catégorie des représentations du groupe symétrique $S_{t}$, lorsque $t$ n'est pas un entier naturel. In: Mehta, V.B. (ed.) Algebraic groups and homogeneous spaces. Proceedings of the international colloquium, Mumbai, India, January 6-14, 2004 209-273 (2007)

9. Drouot, F.r.: Quelques proprietes des representations de la super-algebre der Lie $g l(m, n), \mathrm{PhD}$ thesis (2009)

10. Duflo, M., Serganova, V.: On associated variety for Lie superalgebras. arXiv:0507198v1 (2005)

11. Goetz, G., Quella, T.h., Schomerus, V.: Representation theory of $\mathfrak{s l}$, (2|1). J. Algebra 312(2), 829-848 (2007)

12. Goetz, G., Quella, T.h., Schomerus, V.: Tensor products of $p s l(2,2)$ representations. ArXiv e-prints: arXiv:hep-th/0506072 (2005)

13. Heidersdorf, T.H.: Mixed tensors of the general linear supergroup. J. Algebra 491, 402-446 (2017)

14. Heidersdorf, T.H., Weissauer, R.: Cohomological tensor functors on representations of the general linear supergroup, ArXiv e-prints: 1406.0321, to appear in: Mem. Am. Math Soc (2014)

15. Heidersdorf, T., Weissauer, R.: On classical tensor categories attached to the irreducible representations of the General Linear Supergroup $G L(n \mid n)$. arXiv:1805.00384 (2018)

16. Heidersdorf, T.: On supergroups and their semisimplified representation categories. ArXiv e-prints arXiv: 1512.03420 (2015)

17. Kac, V.: Representations of classical Lie superalgebras. Differ. geom. Meth. math. Phys. II, Proc., Bonn 1977, Lect. Notes Math. 676, 597-626 (1978)

18. Matsumoto, T., Molev, A.: Representations of centrally extended Lie superalgebra psl(2|2). J. Math. Phys. 55(9), 091704 (2014)

19. Quella, T., Schomerus, V.: Superspace conformal field theory. J. Phys. A, Math. Theor. 46(49), 494010 (2013)

20. Serganova, V.: On the superdimension of an irreducible representation of a basic classical Lie super algebra, Supersymmetry in mathematics and physics. UCLA Los Angeles, USA 2010. Papers based on the presentations at the workshop, Februar 2010. Springer, Berlin (2011)

21. Sergeev, A.N.: The tensor algebra of the identity representation as a module over the Lie superalgebras $\mathfrak{G} l(n, m)$ and Q(n). Math. USSR Sb. 51, 419-427 (1985)

22. Weissauer, R.: Monoidal model structures, categorial quotients and representations of super commutative Hopf algebras II: The case $G l(m, n)$, arXiv e-prints (2010)

Publisher's Note Springer Nature remains neutral with regard to jurisdictional claims in published maps and institutional affiliations. 